\title{
Was leistet die subkutane Alttuberkulinprobe zur Erkennung der aktiven Lungentuberkulose bei Erwachsenen?
}

\author{
Von \\ Stabsarzt Dr. Boehalli, \\ z. Zt. Rathonow ; vorher leitender Arzt des Sauatoriums.
}

Die Ansichten über den Wert der subkutanen Tuberkulinprobe gehen immer noch weit auseinander. Das kommt besonders wieder in dem Bericht von Pannwitz über "Tuberkulin in Deutschland" zum Ausdruck, in dem erfahrene Tuberkulinkenner sich in kurzen Sätzen über die Bedeutung des Tuberkulins in therapeutischer und diagnostischer Hinsicht äussern. Die begeistertsten Anhänger des Tuberkulins sind nach wie vor Röpke und Bandelier. Sie halten, wie sie in ihrem Handbuch der Tuberkulose sagen, die subkutane Tuberkulinprobe im Verein mit den sonstigen zur Verfügung stehenden klinischen Untersuchungsmethoden, der Anamnese, der Thermometrie für das souveräne Diagnostikum für die Erkennung der aktiven Lungentuberkulose Erwachsener; sie verdient nach diesen Autoren in allen Zweifelsfällen die weiteste Anwendung, muss aber zur Vermeidung von sogenannten Impfschäden „lege artis" erfolgen. Röpke vertritt diesen Standpunkt auch in dem oben erwähnten Bericht; er ist aber der einzige, der sich so zuverlässig äussert, und nennt die subkutane Tuberkulindiagnostik bei richtiger Methodik unschädlich und leistungsfähig; „treten auf kleinere Dosen prompte Fieber- und Allgemeinreaktionen auf, oder lassen sich im Anschluss daran lokale Herdreaktionen nachweisen, so ist an dem Bestehen einer aktiven Lungentuberkulose nicht zu zweifeln. Tritt bei einem Erwachsenen, der auf Tuberkulose verdächtig ist, auf subkutane Tuberkulindosen 
von $2 / 10,1,5$ und $10 \mathrm{mg}$ keine Reaktion auf, so ist da mit Sicherheit auf Fehlen von aktiven tuberkulösen Prozessen zu schliessen". Kremser bewertet in den letzten Jahren die "positive Reaktion“ nur nach der festgestellten "lokalen Reaktion" an der Stelle der Erkrankung. Petruschky, der ja auch stets für das Tuberkulin eintrat, verwendet zu diagnostischen Zwecken ausschliesslich Alttuberkulin Koch und bevorzugt jetzt die kutane Probe in der Form der von ihm eingeführten flachen Implkreuze. Gebser verwendet Tuberkulin zur Diagnose nur in seltenen Fällen und hält das Resultat nur dann für positiv, wenn Herdreaktion nachweisbar. Nach $\mathrm{Ne}$ is ser geben hohe Reaktionen auf 0,0001 bzw. 0,001 einen gewissen Anhalt für aktive Tuberkulose. $\mathrm{P}$ ischinger wendet diagnostisch neben der Tuberkulin-Hautprobe, die regelmässig gemacht wird, in zweifelhaften Fällen auch die subkutane Probe an. Das Resultat erschien ihm keineswegs absolut zuverlässig und oft schwer zu deuten. Dauernden Schaden hat er bei der Probe nicht gesehen. Auch Ritter wendet die Subkutanprobe nur selten an; sie taugt nicht mehr als die andern". Herdreaktionen, durch die sie natürlich eine gewisse Überlegenheit vor den anderen besitzt, hat er in der Lunge nur selten einwandfrei bekommen. Im neuesten Handbuch der Tuberkulose von Brauer, Schröder und Blumenfeld bespricht Ritter in schöner Arbeit und kritischer Weise eingehend die klinische Bedeutung der Tuberkulinreaktionen. Auch er steht der Subkutanprobe skeptisch gegenüber, gibt die Möglichkeit zu, dass Übertreibungen der Herdreaktion zu stürmischen Einschmelzungen und in Anschluss daran zu einer akuten Weiterverbreitung führen, dass bisher inaktive ruhende Herde zu aktiven und fortschreitenden werden können; er hat auch selbst hie und da kleinere und grössere Schädigungen gehabt, nie aber dauernden Schaden, für den er stets einen Fehler verantwortlich machen will. Henssen, der doch auch über grosse Erfahrungen verfügte, berichtet aber, dass er zweifellose Schädigungen von der subkutanen Reaktion gesehen hat und einen Todesfall an Meningitis auf sie zurückführt. Auch Schultes und $\mathrm{Ziegler}$ äussern sich sehr skeptisch über den diagnostischen Wert des Tuberkulins; Ziegler hat allerdings häufig Herdreaktionen beobachten können. Nach Römer beweist positive Tuberkulinreaktion niemals Tuberkulose-Krankheit, sondern beweist lediglich, dass der Betreffende mit Tuberkulosevirus in Berührung gekommen ist. Der Wert des Tuberkulins für die Erkennung der Tuberkulose als Krankkeit ist sehr gering wegen der ausserordentlichen Verbreitung der Tuberkuloseinfektion. Much sagt, dass die positive Tuberkulinreaktion zur Unterscheidung von aktiver und inaktiver Tuberkulose beim Menschen 
nur benutzt werden kann in der Kindheit und als Herdreaktion. In allen anderen Fällen kann sie die ärztliche Diagnose nur stützen, nicht sichern. Eine negative Reaktion spricht in der Kindheit für Tuberkulosefreiheit; beim Erwachsenen müssen kachektische Zustände und künstlich hervorgerufener Zustand ausgeschlossen werden, ehe sie für Tuberkulosefreiheit verwertet wird. Schudt, der früher diagnostische Einspritzungen mit Alttuberkulin in ausgedehntem Masse gemacht hat, ist immer mehr davon zurückgekommen und wendet sie jetzt nur ganz ausnahmsweise an, da sie ihm bei Lungenaffektionen zur Sicherung der Diagnose und für das therapeutische Handeln keinen wesentlichen Vorteil gebracht. Wolff (Reiboldsgrün) verwendet diagnostisch Tuberkulin so gut wie nie. Sehr ablehnend ist auch Brauer, der die jetzige Tuberkulinära das Stadium ziemlich aussichtslosen, ja fast etwas planlosen Herumexperimentierens nennt. Auch Köhler, Schröder, Ulrici und manche andere haben sich oft skeptisch zur Tuberkulinfrage geäussert.

$\mathrm{Zu}$ diagnostischen $\mathrm{Zwecken}$ habe ich nur das Alttuberkulin Koch benutzt. Schon früher habe ich mich trotz langjähriger und häufiger Anwendung des Tuberkulins während meiner Tätigkeit in Davos, Schömberg und Hohenlychen - meine Erfahrungen erstrecken sich jetzt auf einen Zeitraum von 9. Jahren - nie recht von seinem diagnostischen Wert überzeugen können und insbesondere einwandfreie Herdreaktionen nur selten beobachtet, entgegen den Beobachtungen mancher Autoren, die deren häufiges Vorkommen erwähnen. Auf Grund sorgfältiger Untersuchungen werden sichere Angaben über die Häufigkeit der Herdreaktionen nur selten gemacht; die Angaben von $60 \%$ (Otten) und $90 \%$. (Walterhöfer) hat Ritter nicht bestätigen können; Zablen kann er auch nicht bringen. Aus Zeitmangel kann man bei einem grossen Material in den Heilstätten auch nicht so sorgfältig auf jede Herdreaktion achten und die Beobachtung während der Reaktion durch mehrere Untersucher (Chefarzt oder Assistenten) erhöht die Unsicherheit, und ich glaube Ritter gern, dass Fälle, in denen sich alle untersuchenden Ärzte über eine bestehende Herdreaktion einig waren, nicht allzu häufig gewesen sind. Auch wir hatten in den verschiedenen Anstalten meiner Tätigkeit die einzelnen Reaktionsfälle nicht nach bestimmter Methodik genau durchuntersucht und registriert, sondern meist wurde auf der Höhe der Reaktion mal nachgehorcht; es ist klar, dass man hierbei Täuschungen ausgesetzt ist, meist wohl in dem Sinne, dass man Herdreaktionen häufiger annimmt, als sie wirklich vorhanden sind. Den früheren Befund hat man bei der Untersuchung, die bei der Visite meist nur kurz ist, nicht gegenwärtig, man glaubt, eine Herd- 
reaktion erwarten zu müssen, und man hört sie. So werden in vielen Fällen die Angaben über die Häufigkeit der Herdreaktionen wohl nur schätzungsweise entstanden sein ohne sichere Grundlage, da keine -Aufzeichnungen vorlagen. Die Beobachtungen während der Tuberkulinreaktion müssen aber, wenn sie der Kritik standbalten sollen, ausserordentlich sorgfältig sein und erfordern häufige Untersuchungen, die natürlich zu den verschiedensten Zeiten vor, während und nach der Reaktion, und immer nur von demselben Untersucher vorgenommen werden müssen. Denn nur die Befunde sind vergleichbar, die ein und derselbe Untersucher mit derselben Untersuchungsmethode erhalten hat, und nur so ist eine genaue Kontrolle möglich. Seit der Leitung des Mittelstandssanatoriums in Hohenlychen, das zur Aufnahme von lungenkranken Frauen und erwachsenen Mädchen dient, habe ich mich nun besonders dieser genauen Kontrolle bei Anwendung der probatorischen Tuberkulinimpfung unterzogen und bis zum Ausbruch des Krieges, durch den infolge Zeitmangels die Fortsetzung der zeitraubenden Untersuchungen unmöglich wurde, 55 Fälle sorgfältigst nach bestimmter Methodik beobachtet. Ich ging dabei folgendermassen vor: Nachdem bei der Aufnahme, wie üblich, eine genaue Vorgeschichte und genauer Lungenbefund erhoben, wurden die Patienten vor Einleitung der Tuberkulinprobe zunächst 14 Tage beobachtet, um schon den Einfluss der hygienisch-diätetischen Kur kennen zu lernen und etwaige Änderungen durch die Probeimpfung richtiger beurteilen zu können. Die Patienten wurden täglich $6 \mathrm{mal}$ gemessen, und zwar Mundmessung bei Ruhelage; Liegekur wurde 6 Stunden am Tage innegehalten; nach einigen Tagen aber meist schon 1-2 Stunden Spaziergang verordnet. Da die Beobachtung nach 14 Tagen in diesen Fällen noch keine Klarheit über die Aktivität des Prozesses gab, wurde die subkutane Tuberkulinprobe angestellt. Die Grundbedingung, dass nur fieberfreie Fälle gespritzt werden dürfen, war natürlich erfüllt; die Mundmessung war meist unter 37, nie über 37.3. Ich wählte die in Davos übliche Gabenfolge: 0,$1 ; 0,5$; $1 ; 5 \mathrm{mg}$. Wir gingen in Davos allerdings bis $10 \mathrm{mg}$; nach meinen früheren Erfahrungen ist diese Enddosis aber entschieden zu hoch und kommt für den praktischen Wert nicht in Betracht, da auf $10 \mathrm{mg}$ wohl auch Gesunde reagieren, zum mindesten aber oft inaktive Herde aktiv werden. Häufig wurde mit Karbelkochsalzinjektionen begonnen bei nervösen, ängstlichen Patientinnen, um eine psychische Temperatursteigerung auszuschalten, wie sie von $\mathrm{Köhler}$ und Weihrauch beobachtet ist und mir auch von früher her bekannt war; mitunter wurde auch eine Karbolkochsalzeinspritzung eingeschaltet. Als Verdünnungsflüssigkeit diente eine Lösung von: Acid. 
carbol. 2,5, Natr. chlorat. 3,0, Aquae destill. ad 500,0, die steril in der Apotheke angefertigt wurde. Man gebraucht für die diagnostische Probe ja nur 2 Verdünnungen, die man sich am besten selbst in folgender einfachster Weise bereitet: I. Lösung: 0,1 ccm Alttuberkulin mit 9,9 ccm der Karbolkochsalzlösung mischen; dann sind in einer Spritze ( $=1 \mathrm{ccm}) 10 \mathrm{mg}$ Alttuberkulin, in einem Teilstrich (=1/10 ccm) der Spritze $1 \mathrm{mg}$ Alttuberkulin. II. Lösung: $1 \mathrm{ccm}$ der ersten Lösung mit $9 \mathrm{ccm}$ Karbolkochsalzlösung mischen, dann sind in einer Spritze $1 \mathrm{mg}$, in einem Teilstrich der Spritze 0,1 mg. Beide Lösungen werden in dunklen Flaschen mit weitem Halse kühl aufbewahrt und halten sich mindestens 14 Tage lang. Die Einspritzungen wurden zweimal wöchentlich in den Rücken gemacht, da die Injektionen in den Vorderarm zu schmerzhaft sind und unangenehme Stichreaktionen ergeben können; es wurde morgens um 8 Uhr gespritzt, um die nach 6-10 Stunden auftretende Reaktion bei der Abendvisite schon beobachten zu können. Es kam nicht vor, dass Reaktionen, die in die Nacht fielen, übersehen wurden, meist ist am Abend schon der Beginn der Reaktion und die Patienten waren dann angewiessen auch nachts $z u$ messen, was sie schon infolge bestehender Schlaflosigkeit, vielfach von selbst und öfters als nötig war, taten. Die subjektiven Angaben und der objektive Lungenbefund wurden stets genau festgelegt; während der ganzen Dauer der Reaktion wurden von mir wiederholte eingehende Untersuchungen vorgenommen. Nur wirklich einwandfreie Herdreaktionen bezeichnete ich als positiv, die mir, soweit es sich überhaupt ermöglichen lässt, nach dieser systematischen Methodik kaum entgehen konnten. Dass trotzdem immer das persönliche Ermessen eine gewisse Rolle spielt, gebe ich gern zu; der Fehler ist jedenfalls so am kleinsten. Selbstverständlich werden kleine Herdreaktionen, zumal im Innern des Körpers stets unbemerkt bleiben und sich nach den üblichen Untersuchungsmethoden nicht nachweisen lassen; damit ist aber auch die praktische Wertlosigkeit solcher kleinen Reaktionen von vornherein erwiesen.

Auf diese Weise sind die nachfolgenden Untersuchungstabellen entstanden, die die Ergebnisse der 55 probatorischen Alttuberkulinimpfungen wiedergeben. 


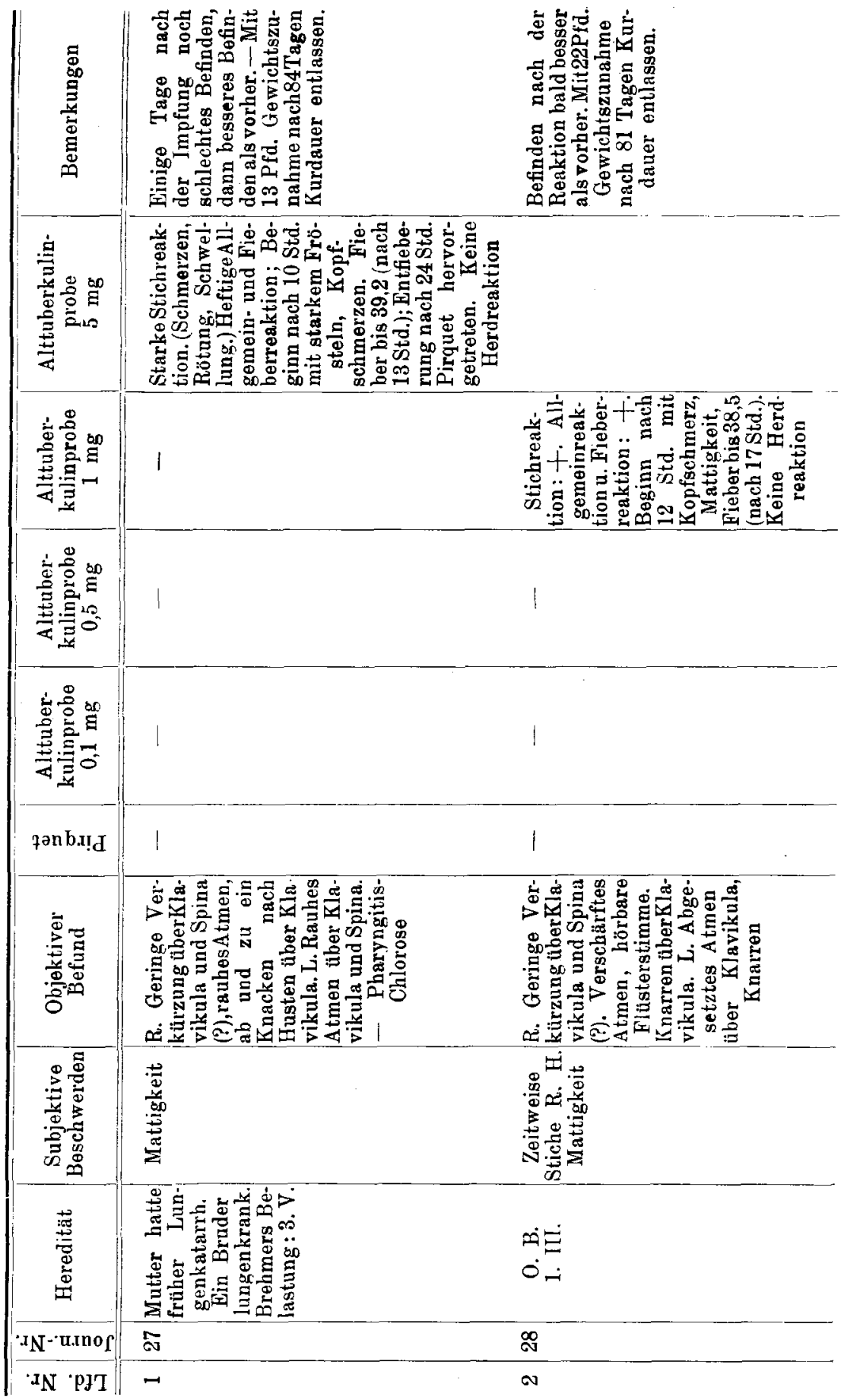



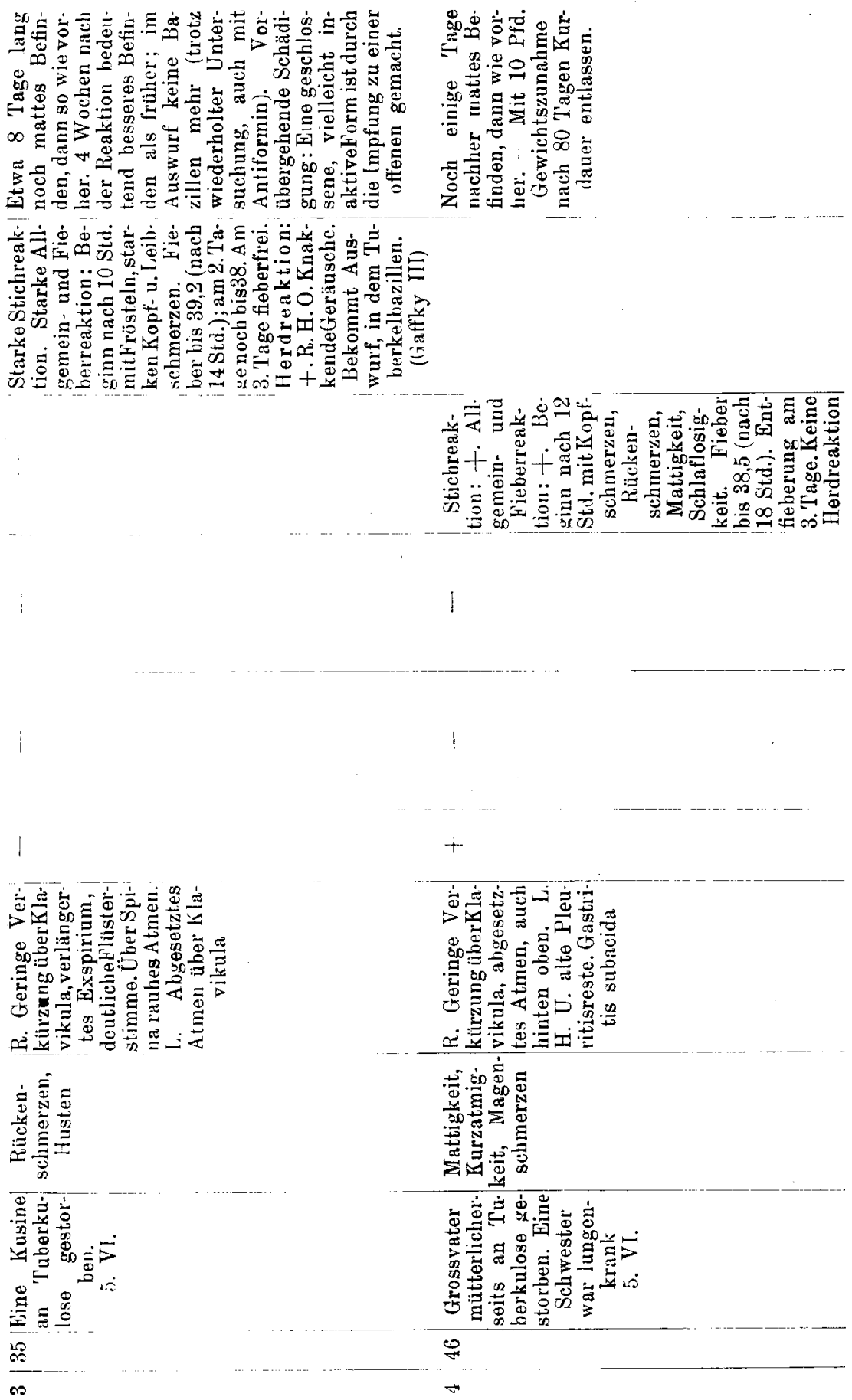

$+$

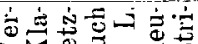

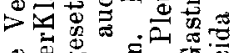

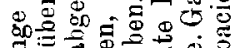

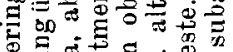

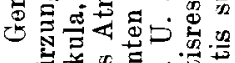

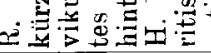

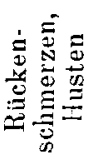

茄荡

ब.

300

焉

究幽志

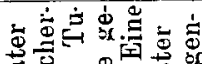

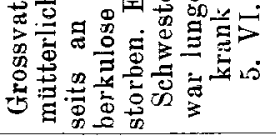

\%

$\leftrightarrow$

Beiträge zur Klinik der Taberknlose. Bd. XXXV. H. 2. 


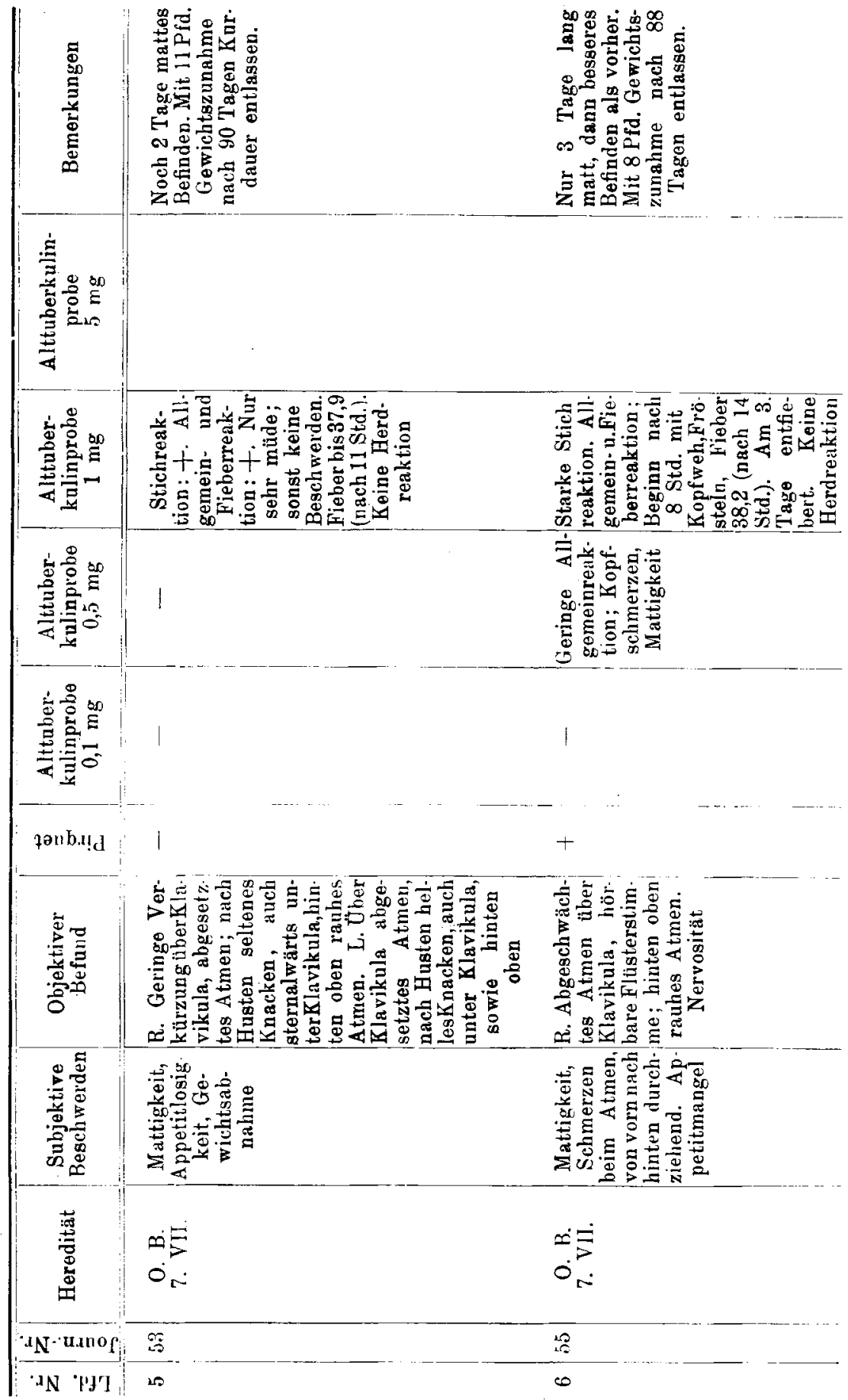



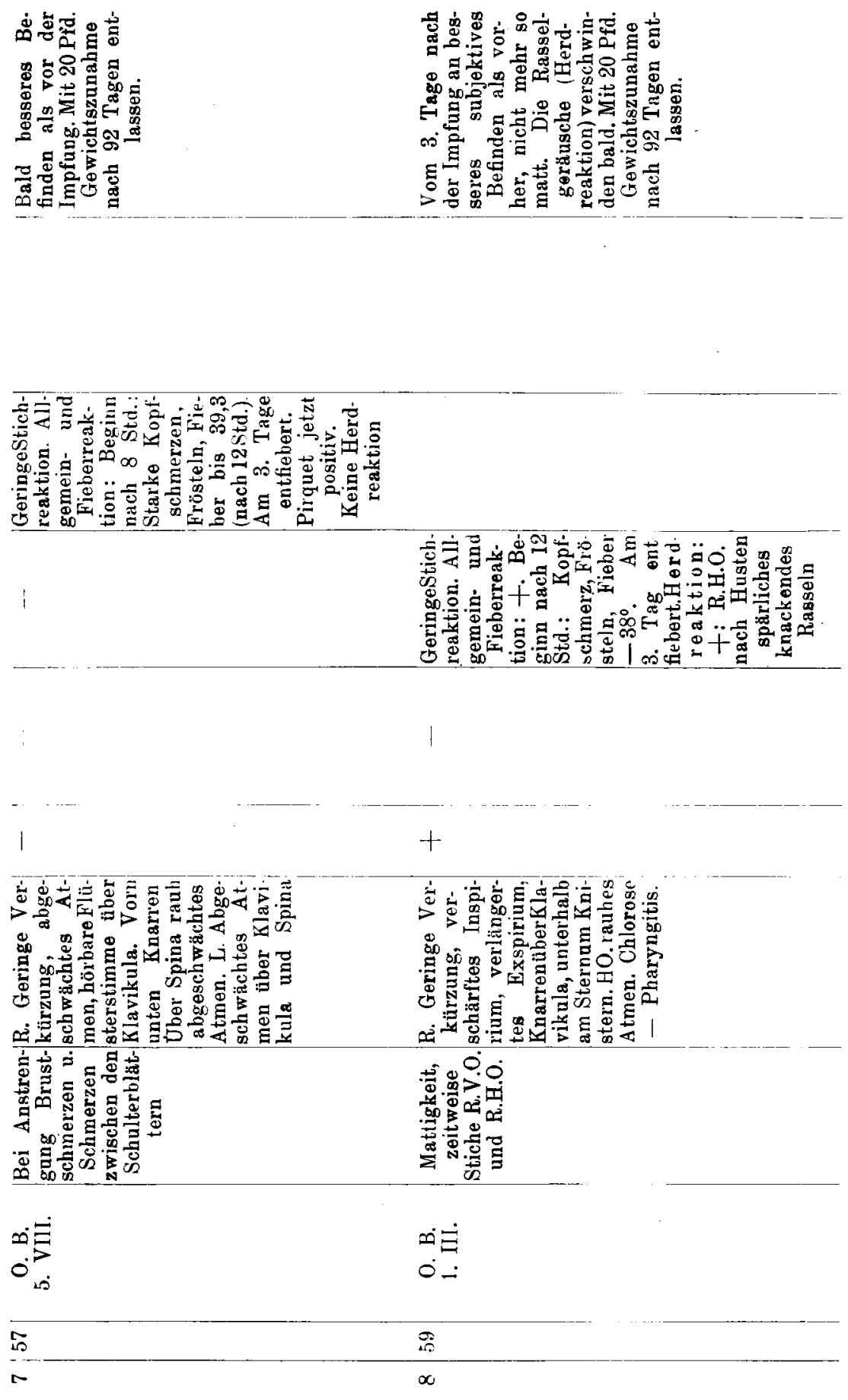


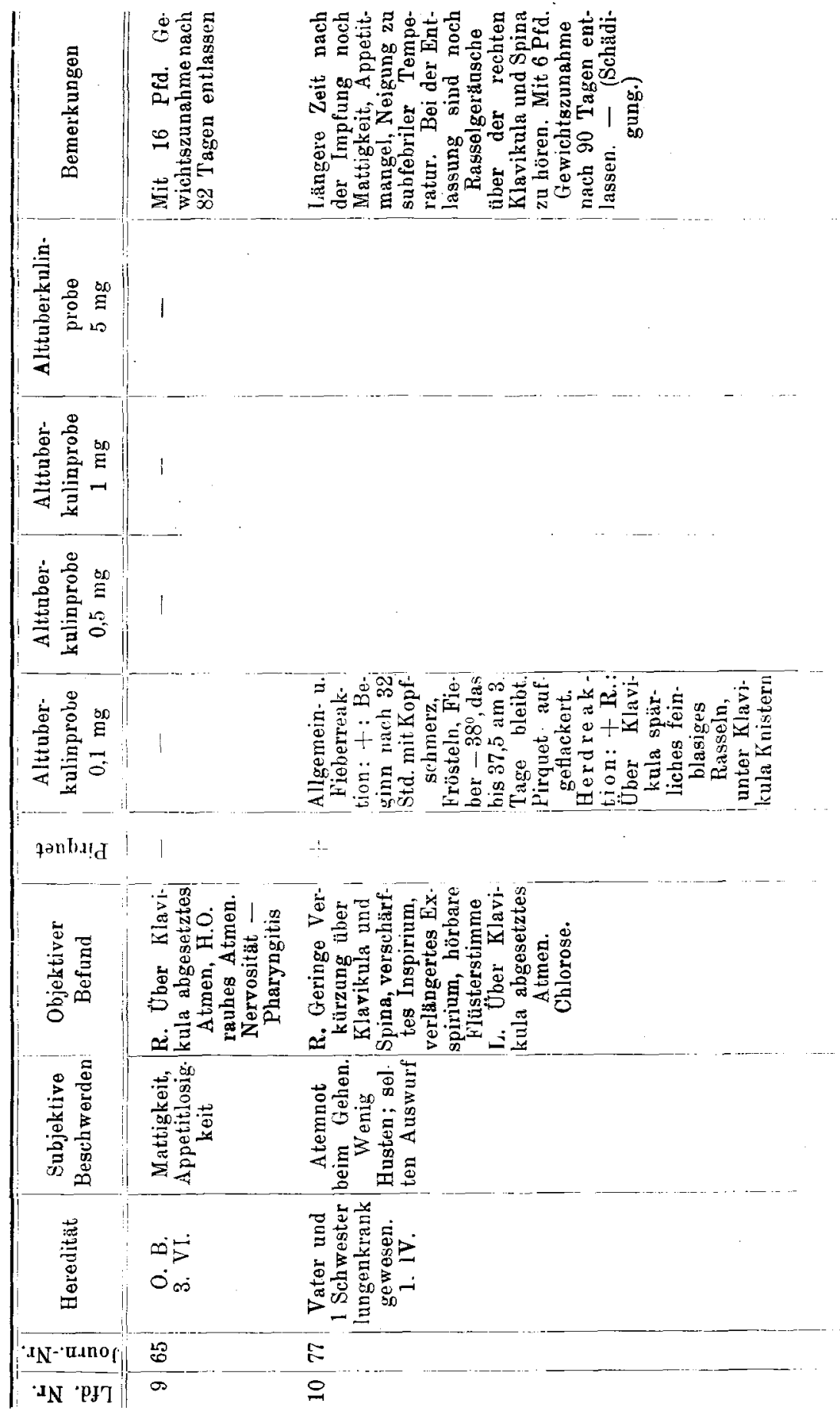



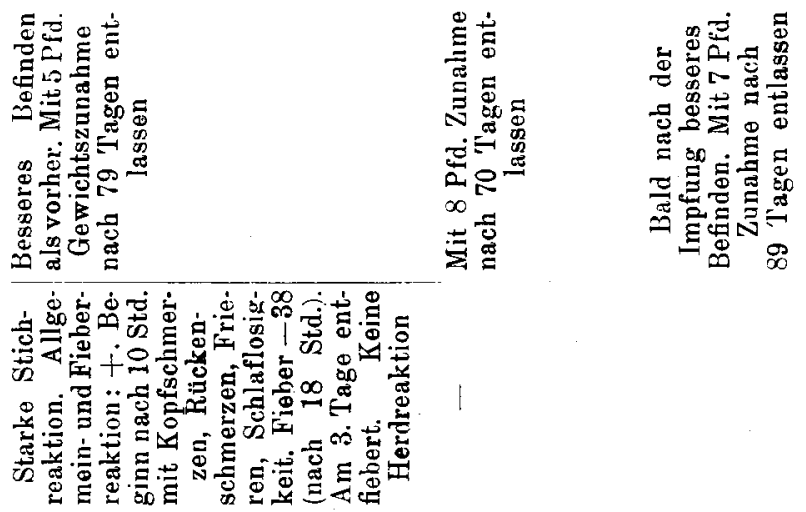

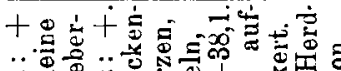

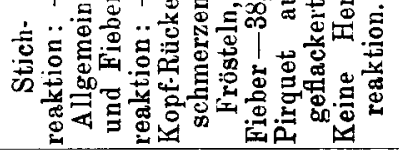

\begin{tabular}{|c|c|c|}
\hline I & + & + \\
\hline 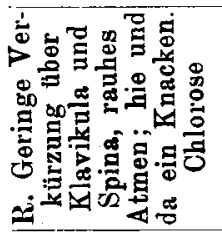 & 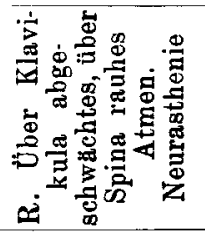 & 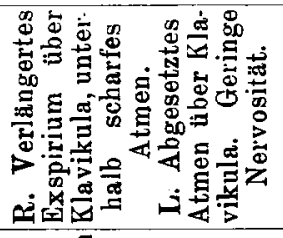 \\
\hline 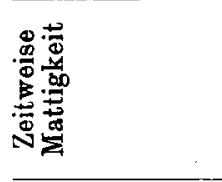 & 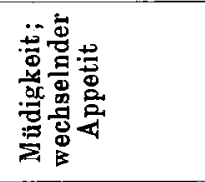 & 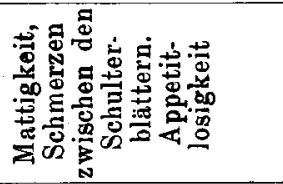 \\
\hline 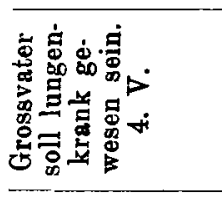 & 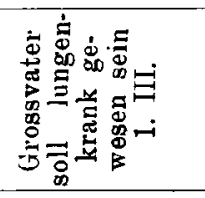 & 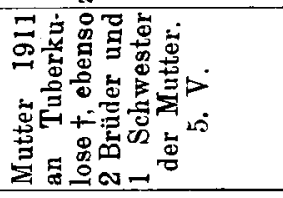 \\
\hline$\Xi$ & 28 & P \\
\hline$\Rightarrow$ & 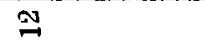 & $\stackrel{\infty}{\rightarrow}$ \\
\hline
\end{tabular}




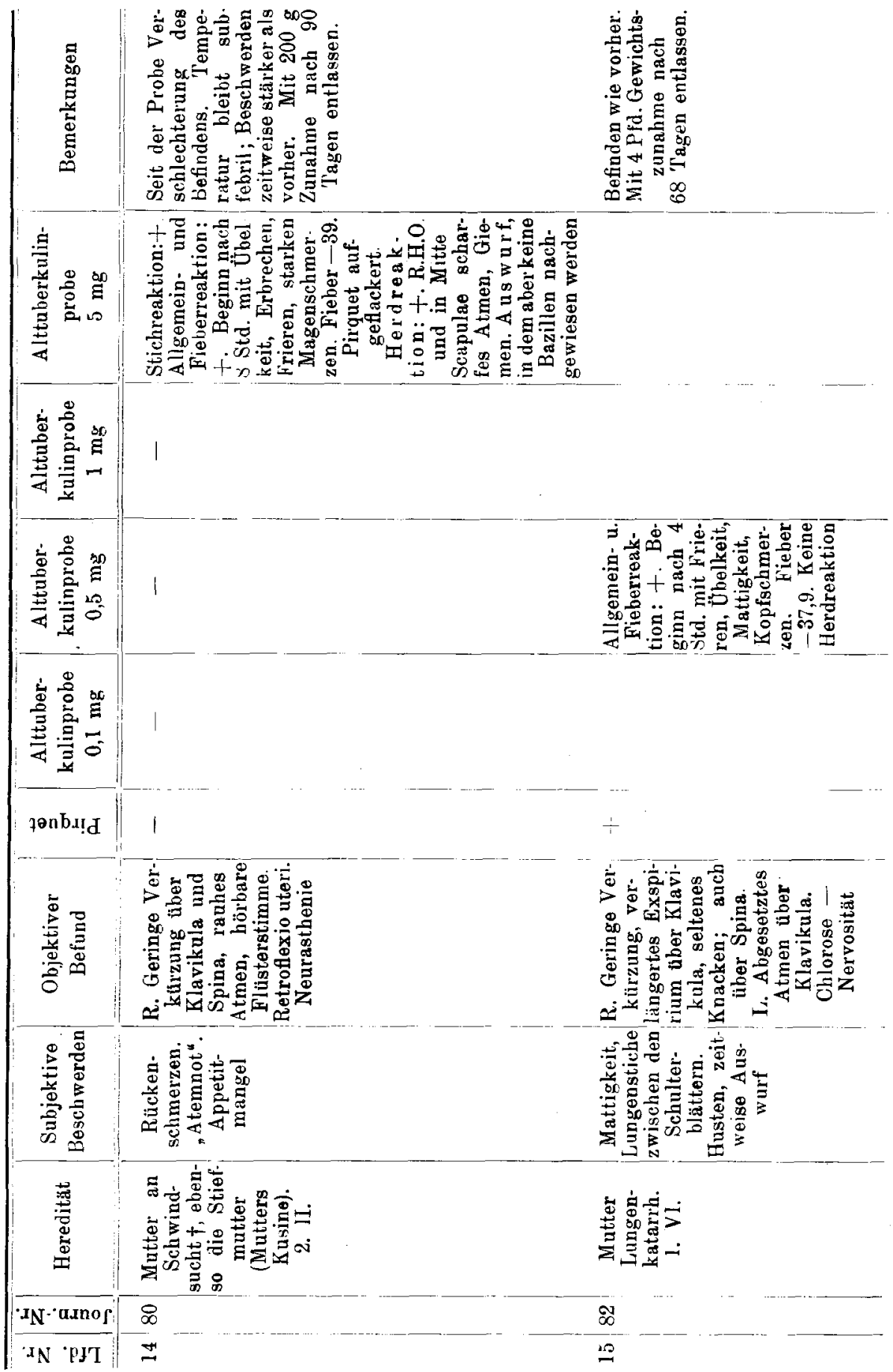



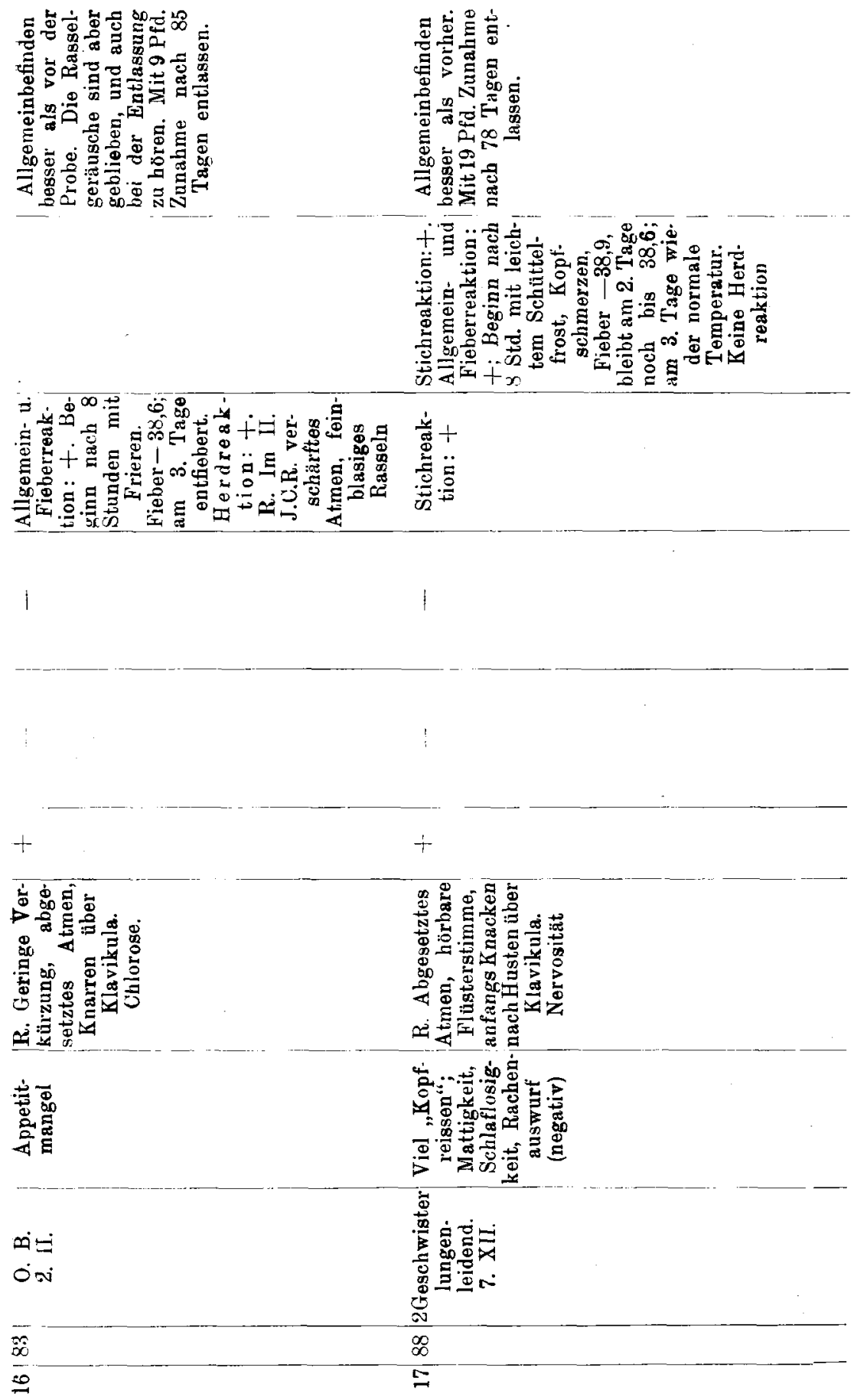


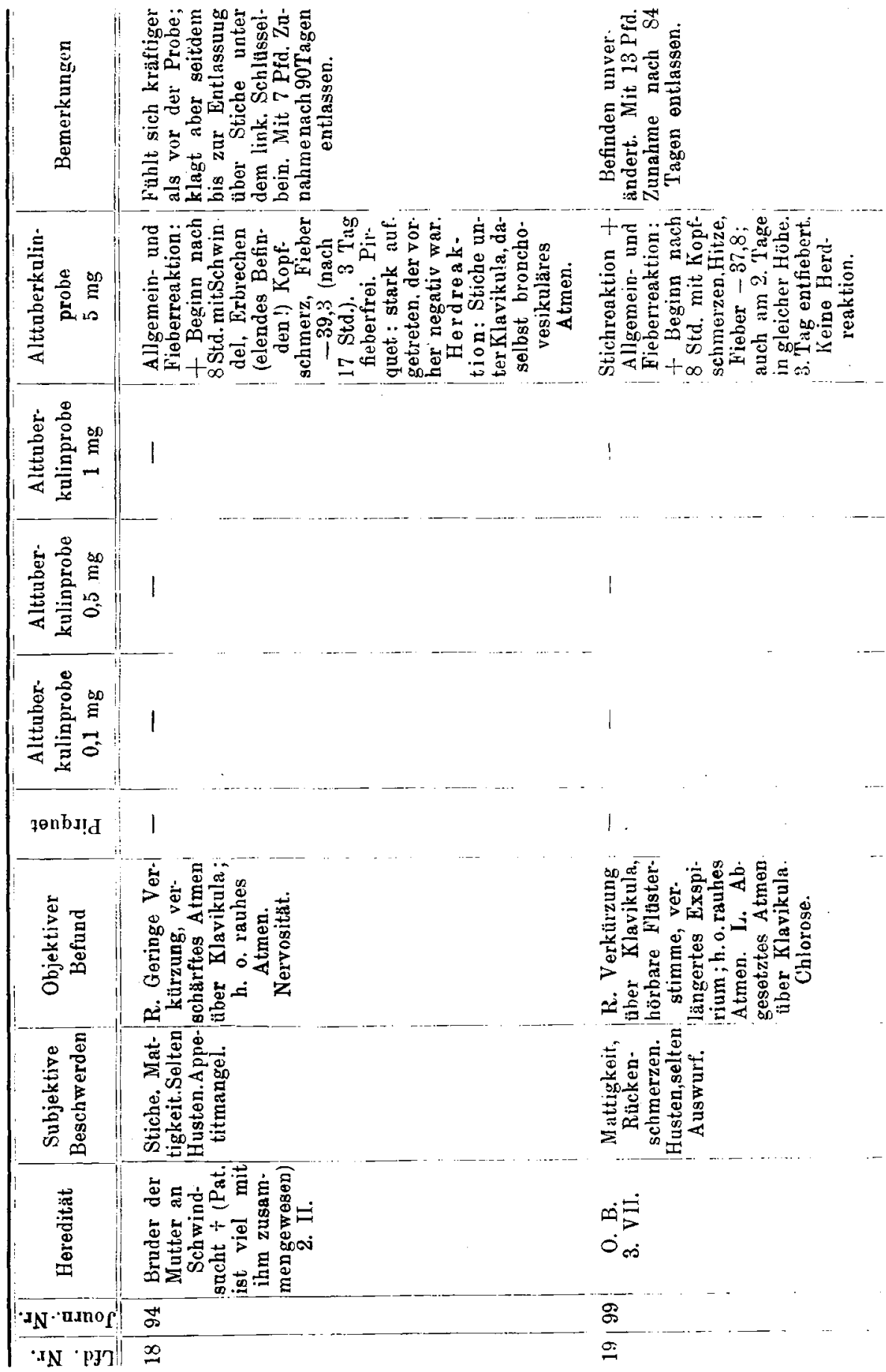



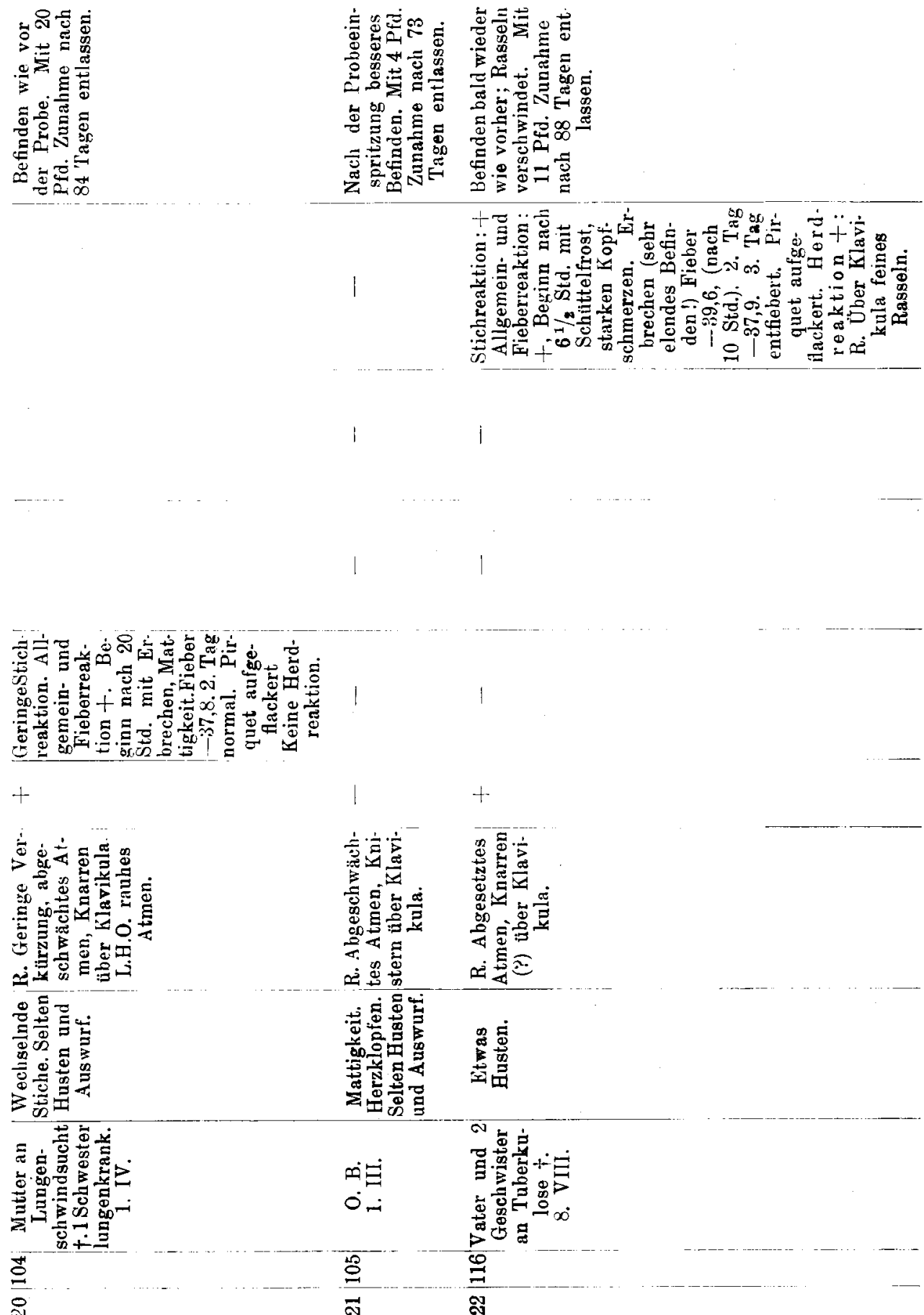

워
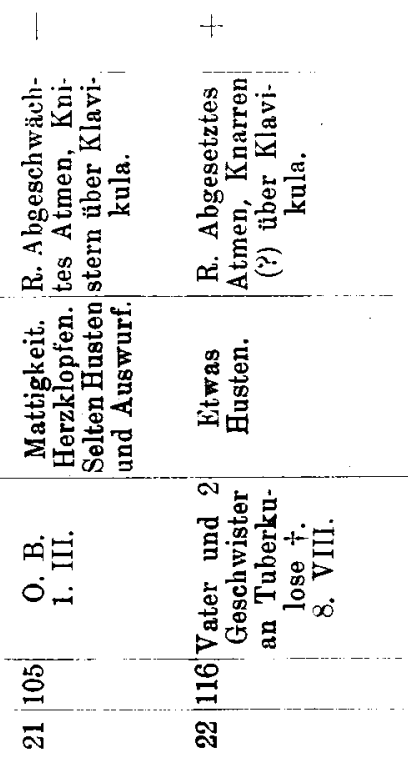


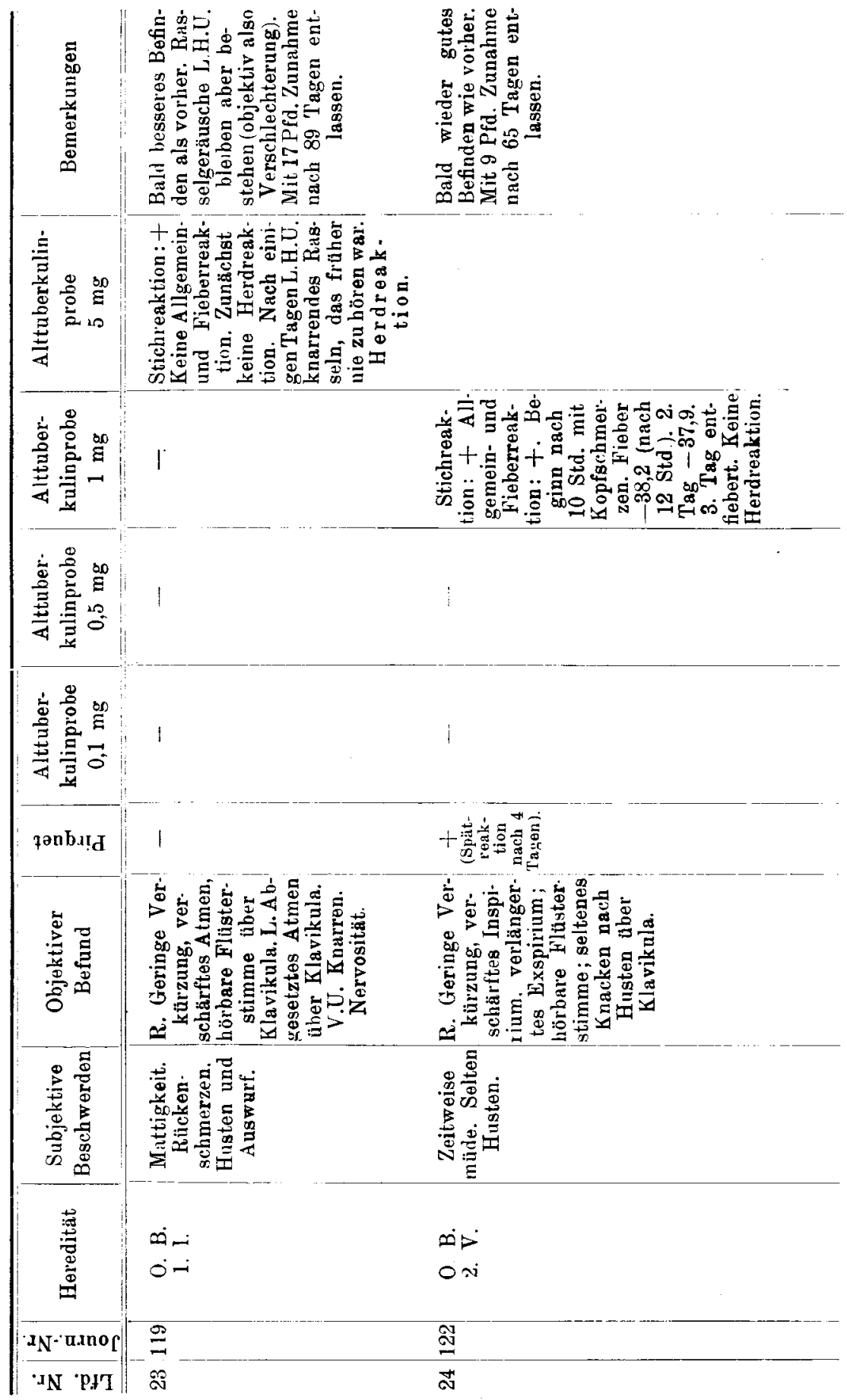



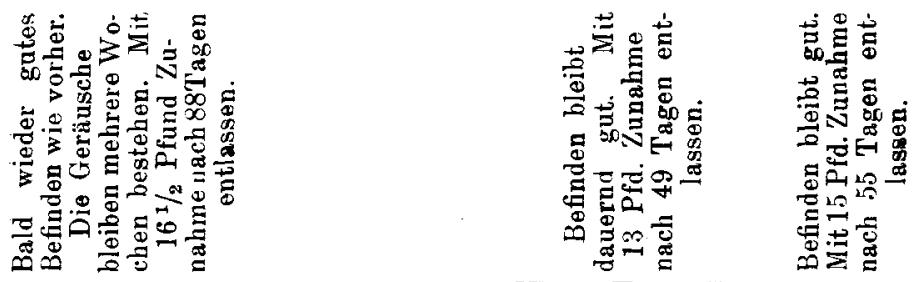

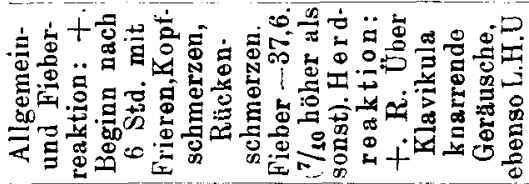

।

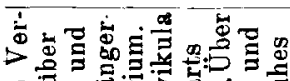

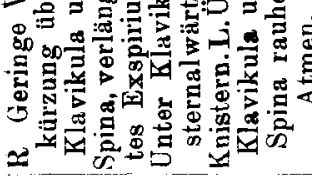

泀焉

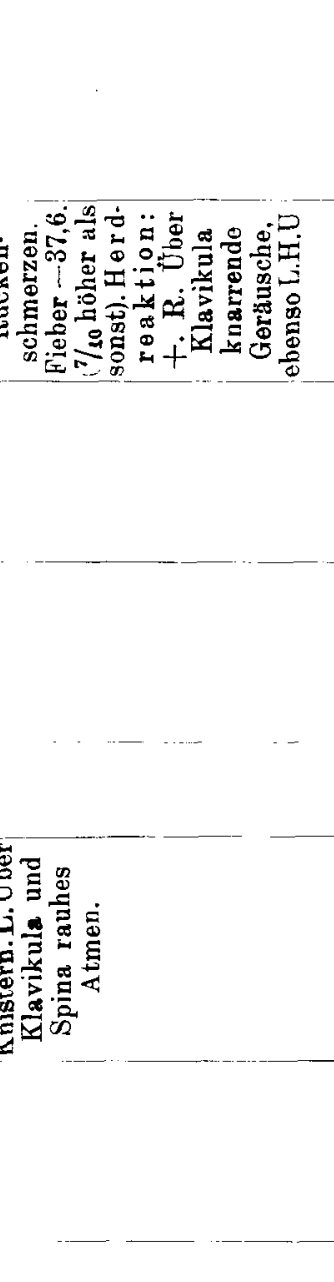

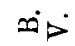

$\dot{0} \dot{0}$

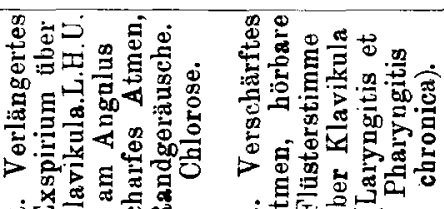

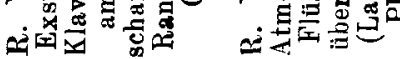
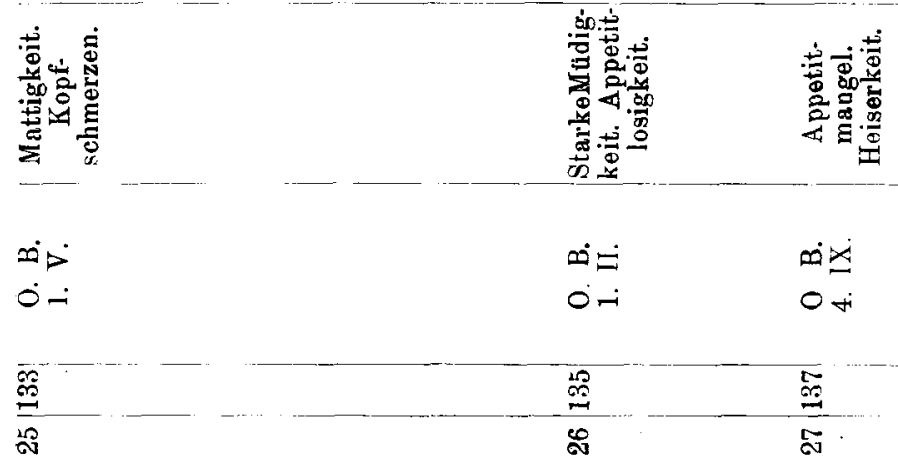


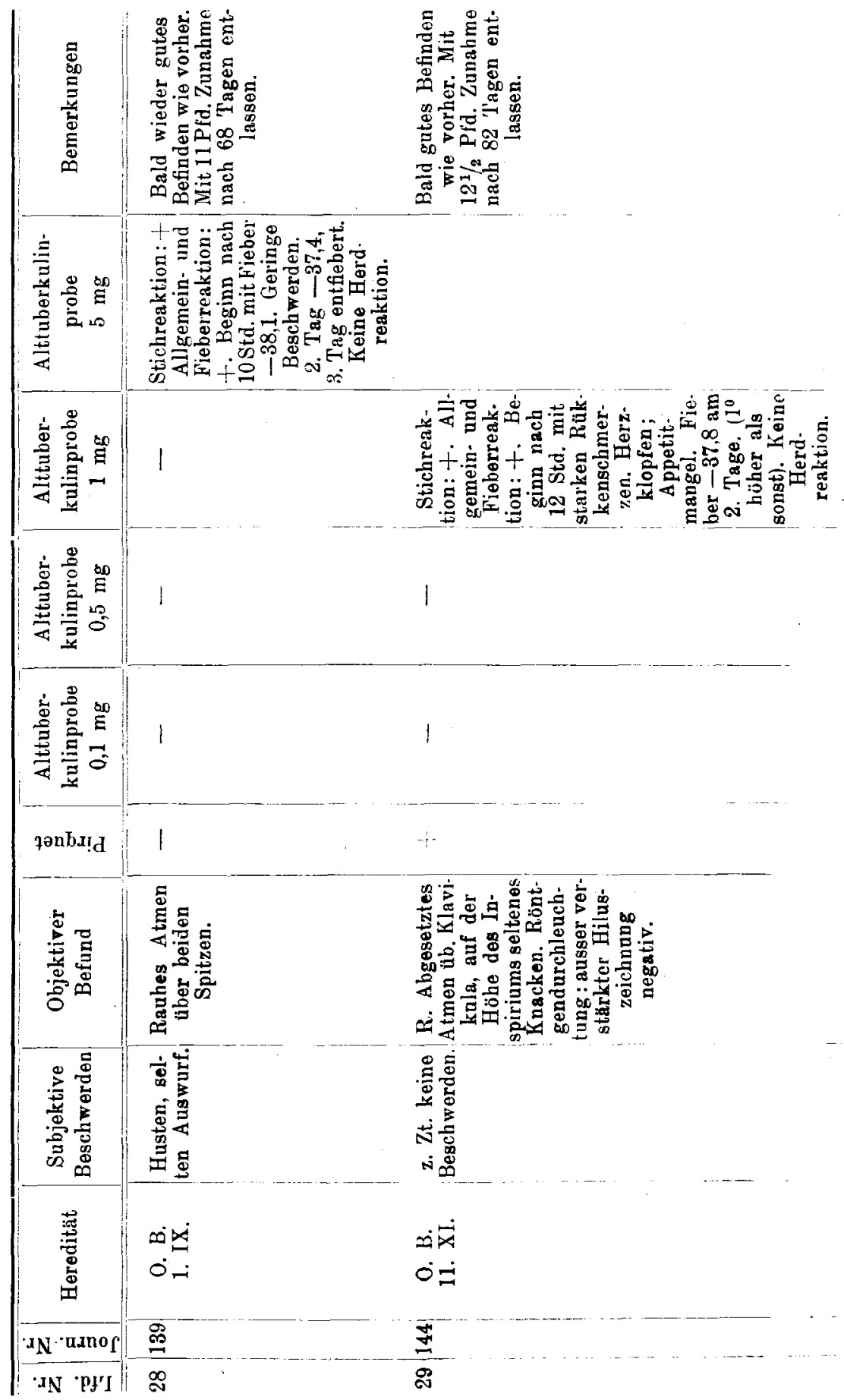



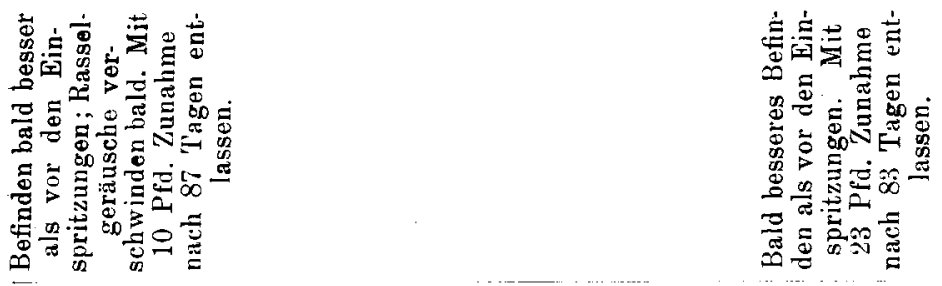

†.

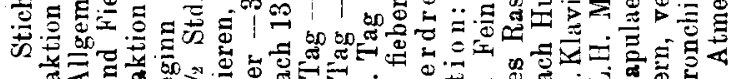

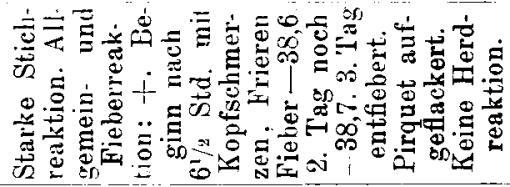

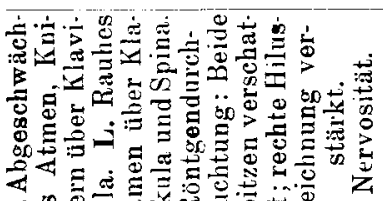

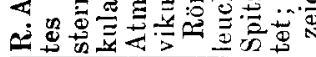

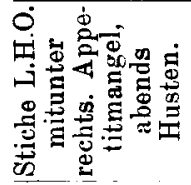

๑๋

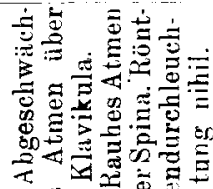

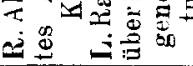
i.

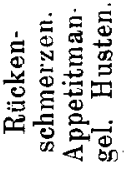

$\frac{\overline{0}}{\frac{1}{8}}$ 羿 


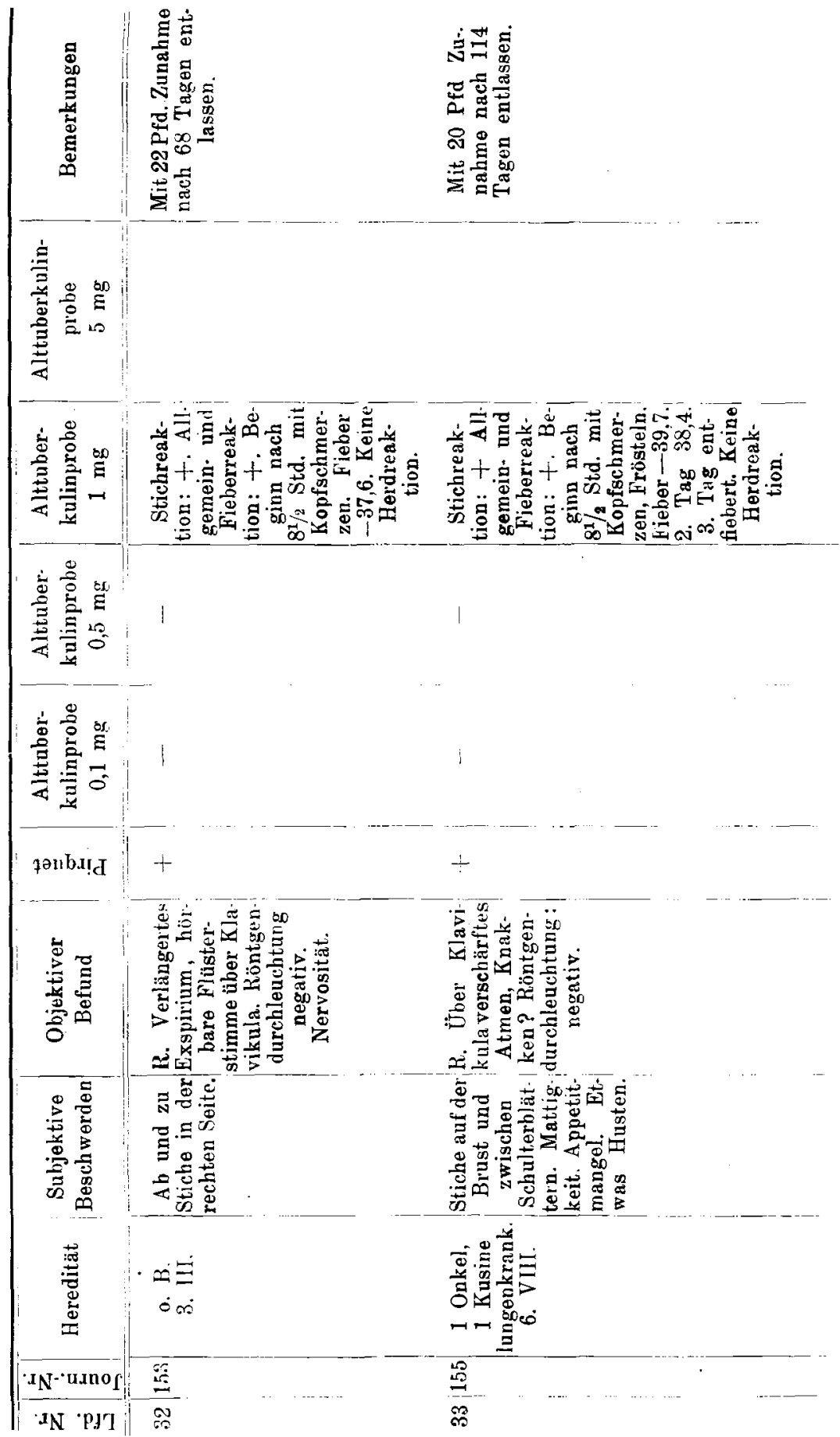



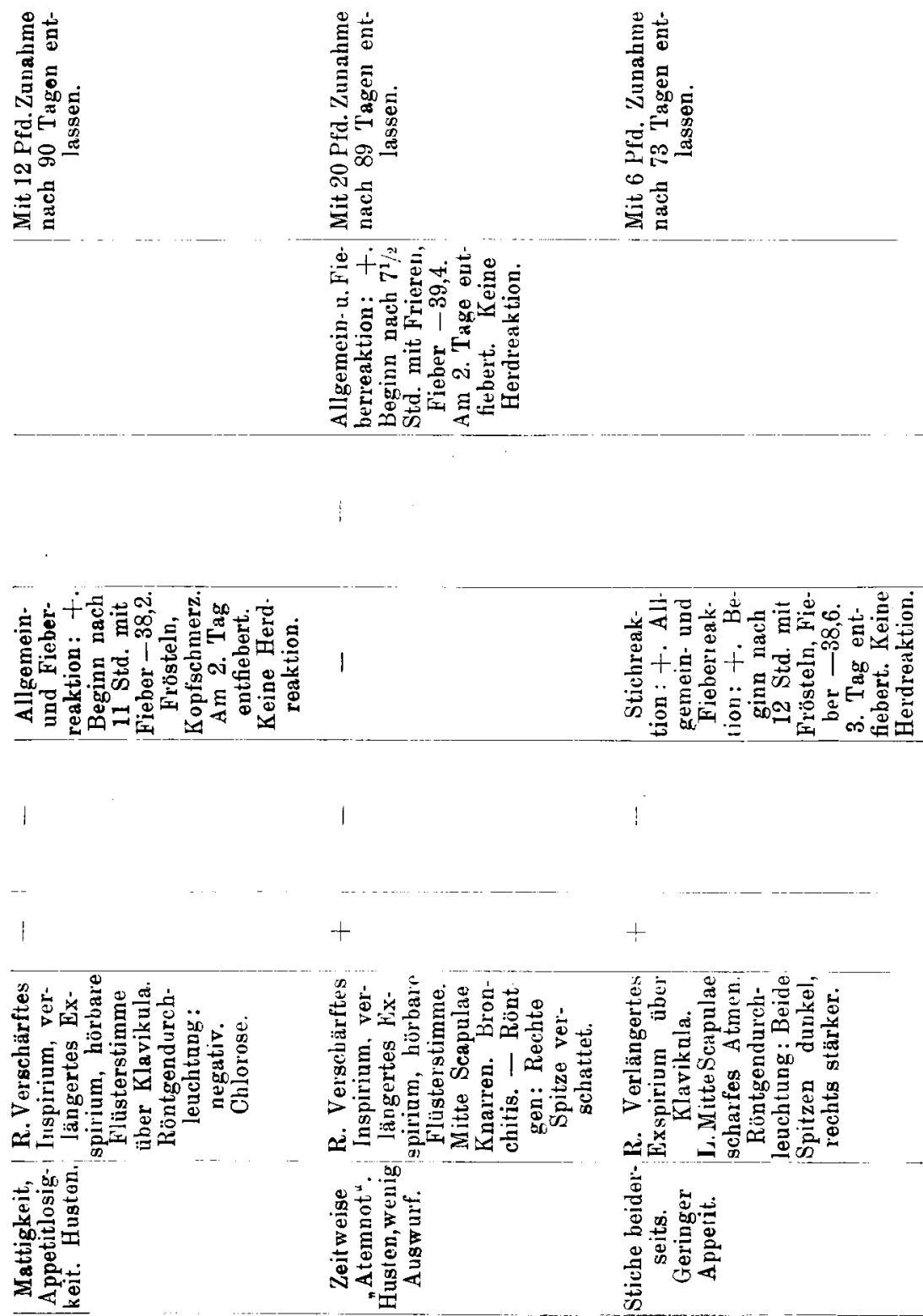
$\infty$
$x=$
$\infty=$
0
0.5
$\dot{0}+$

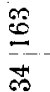
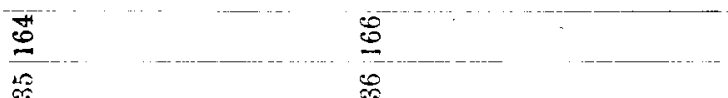


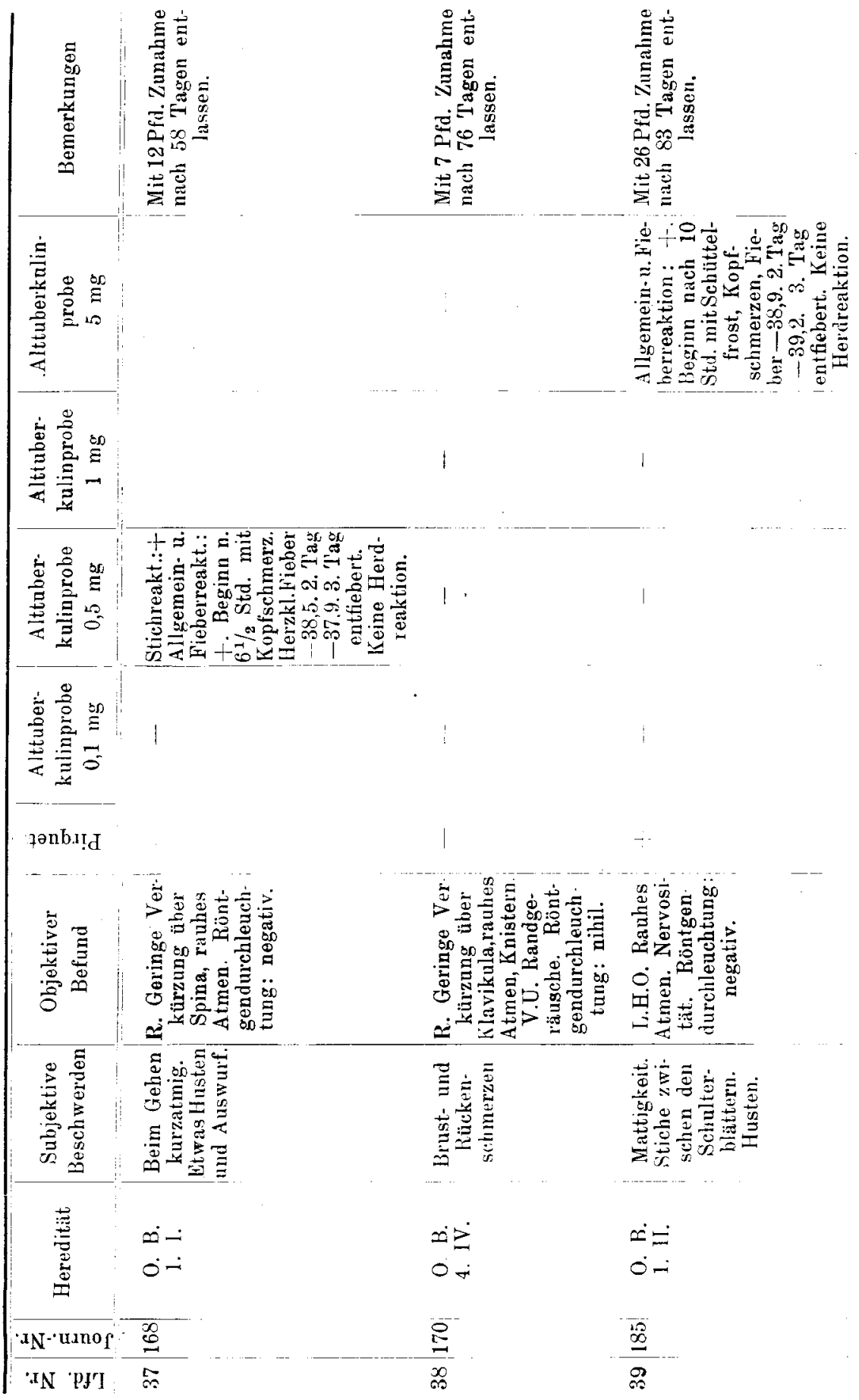



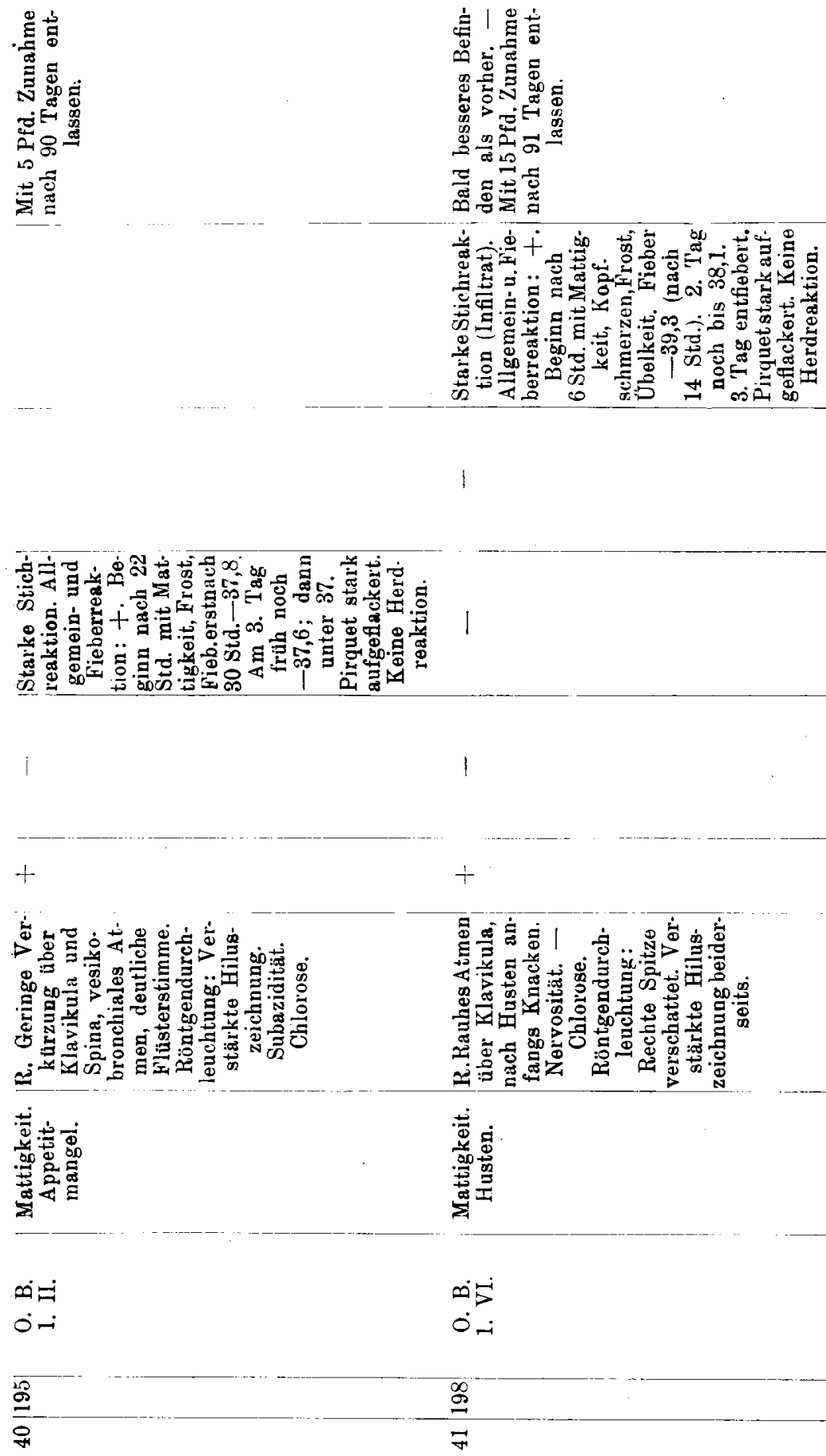

Beitrăge zur Klinik der Taberkalose. Bd, XXXV. H, 2. 


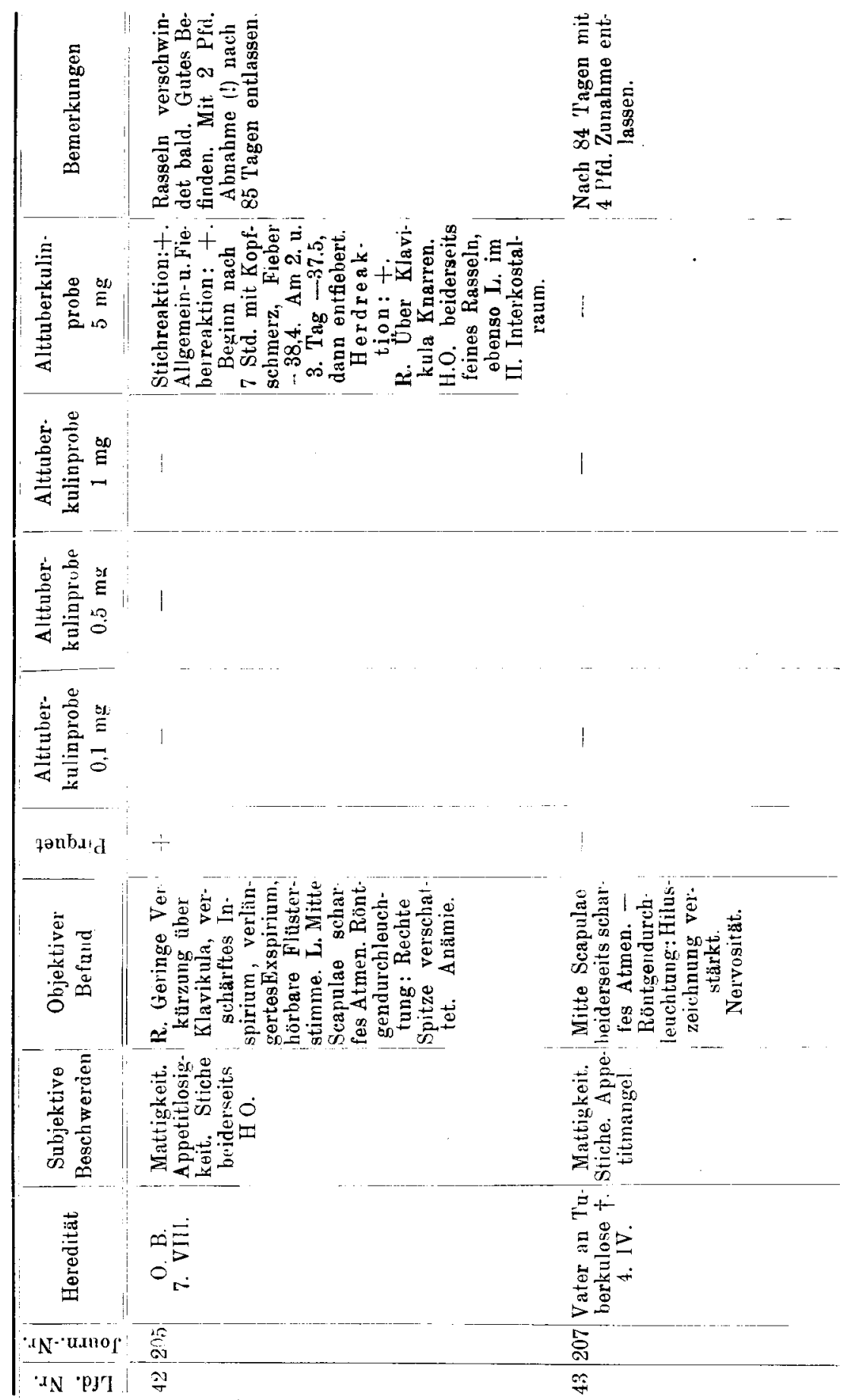



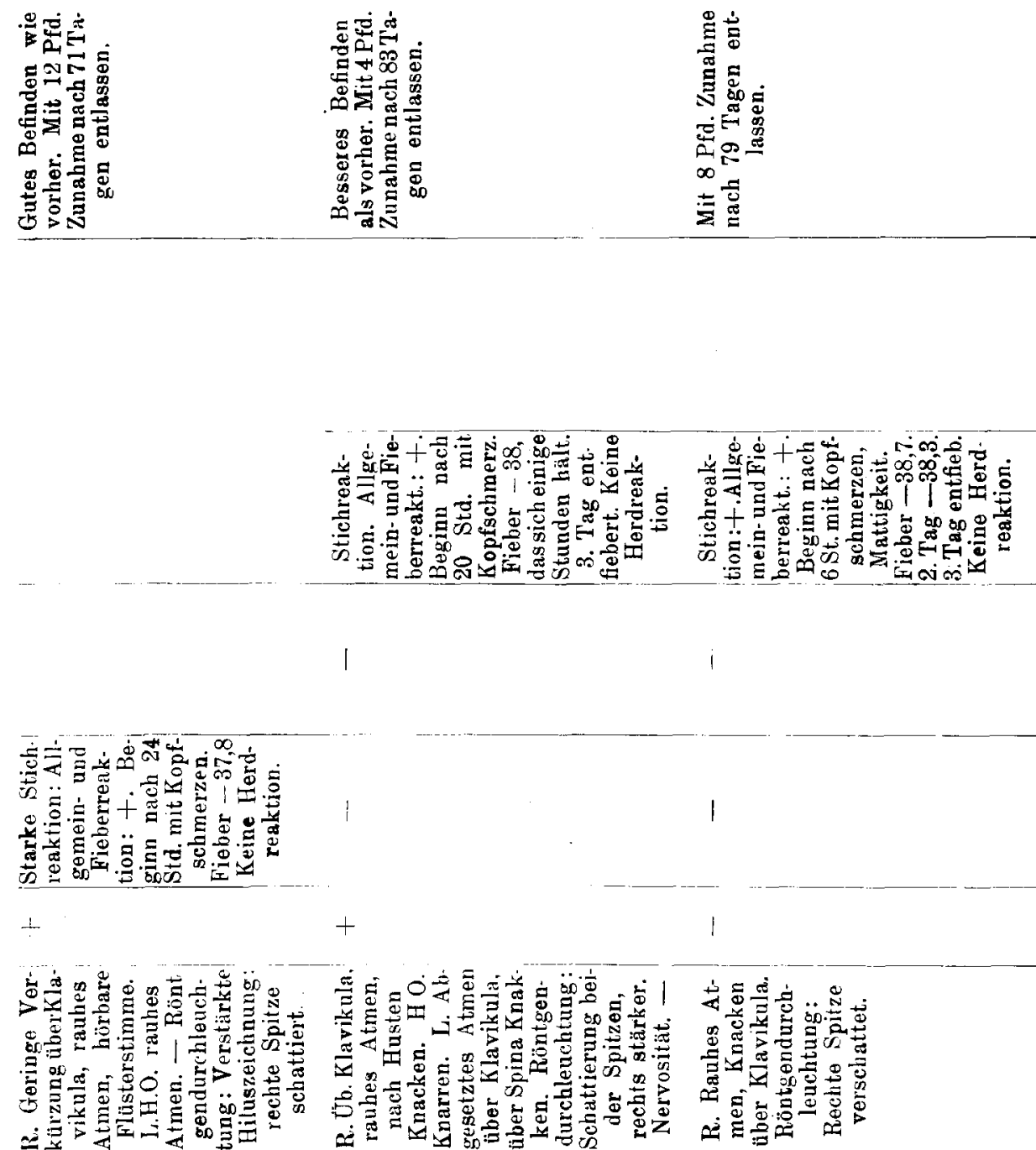

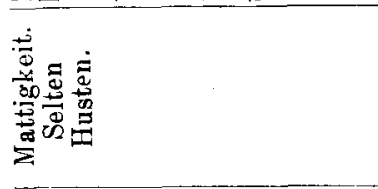

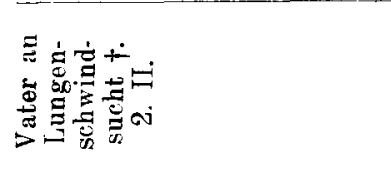
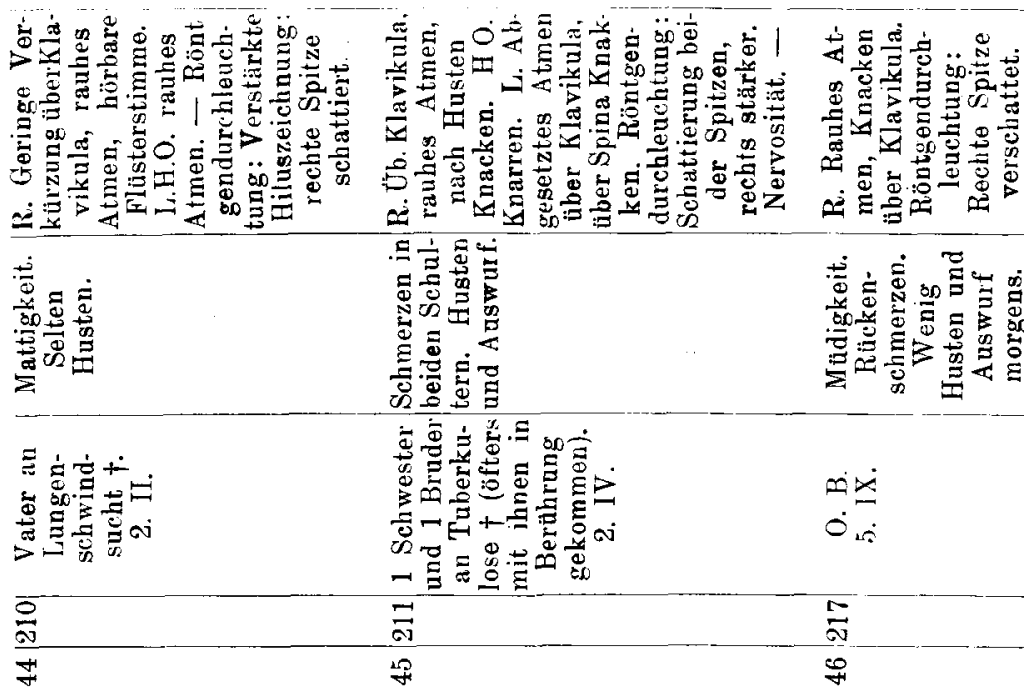

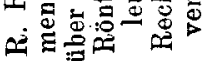

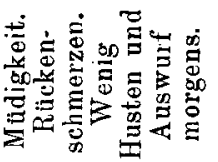

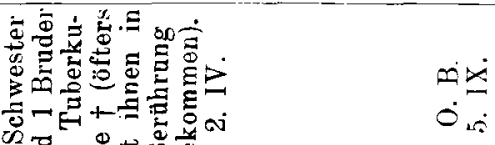

क人

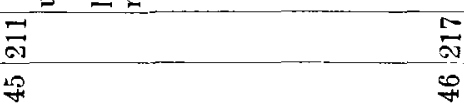




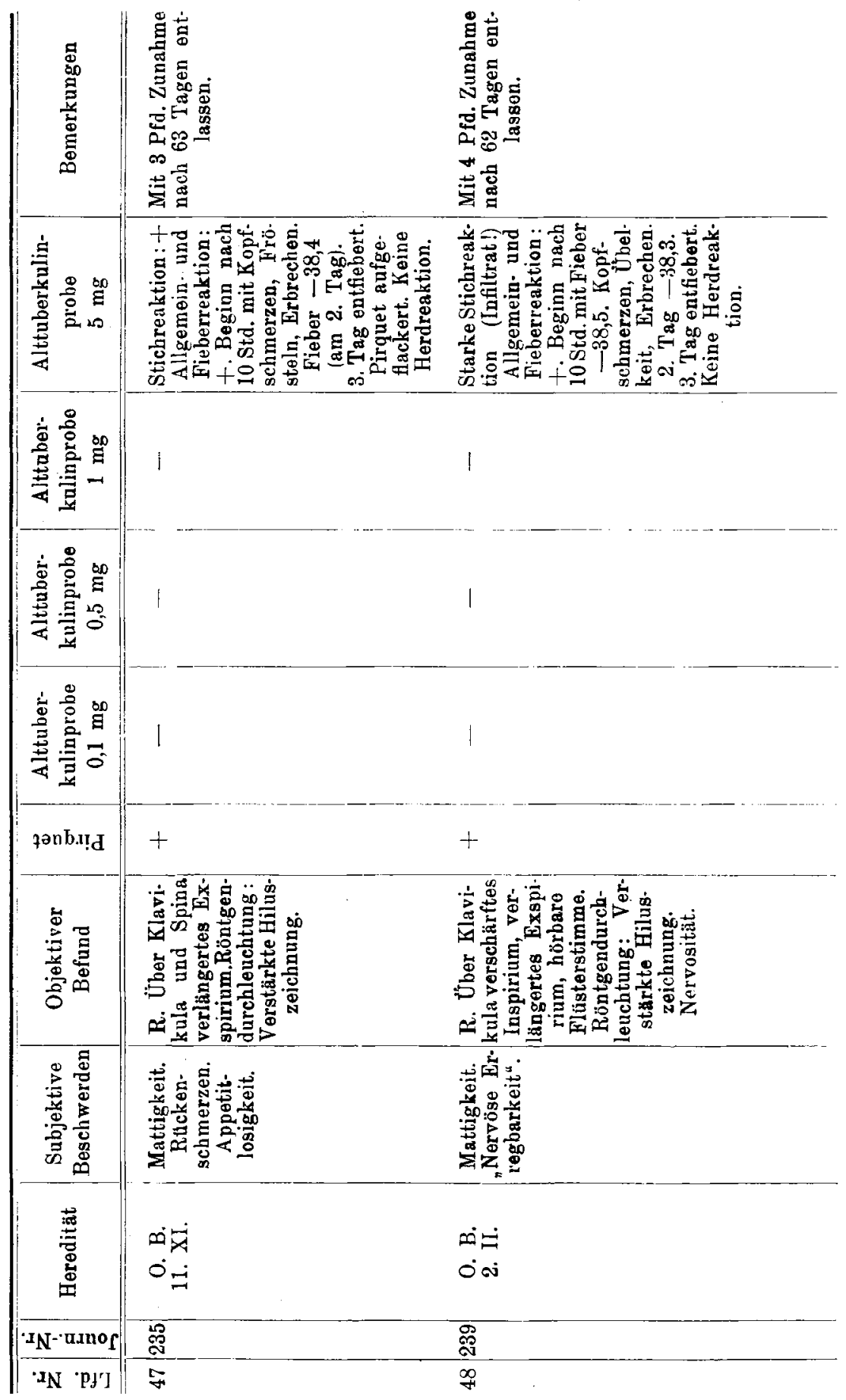



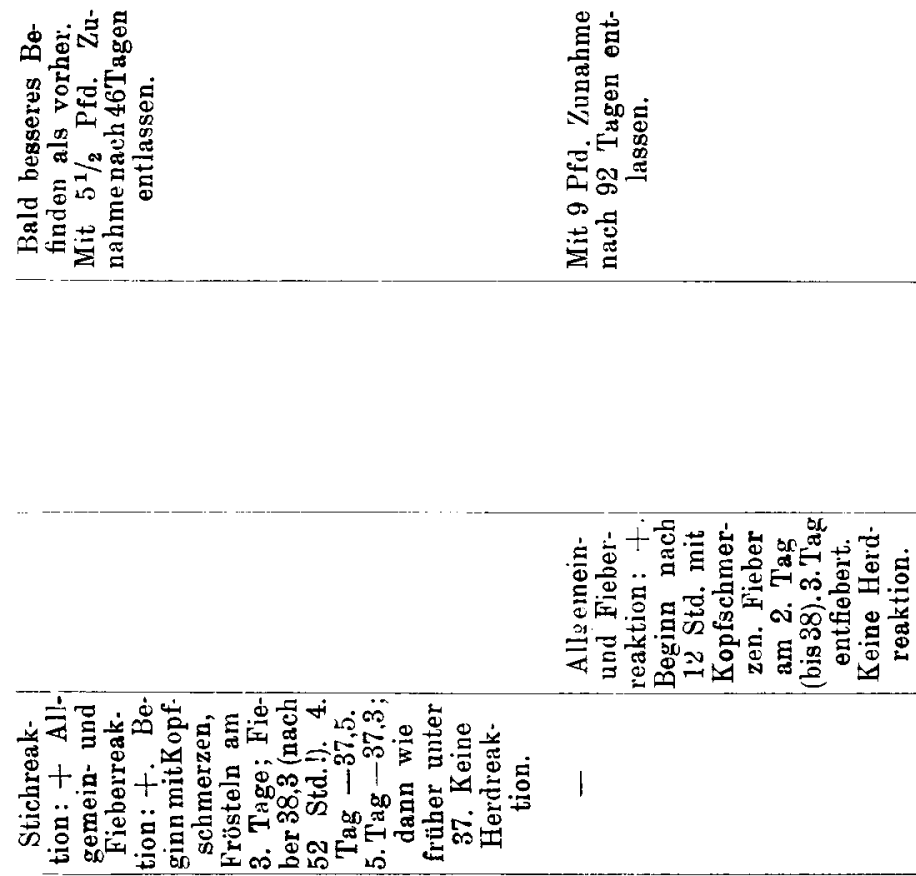

1

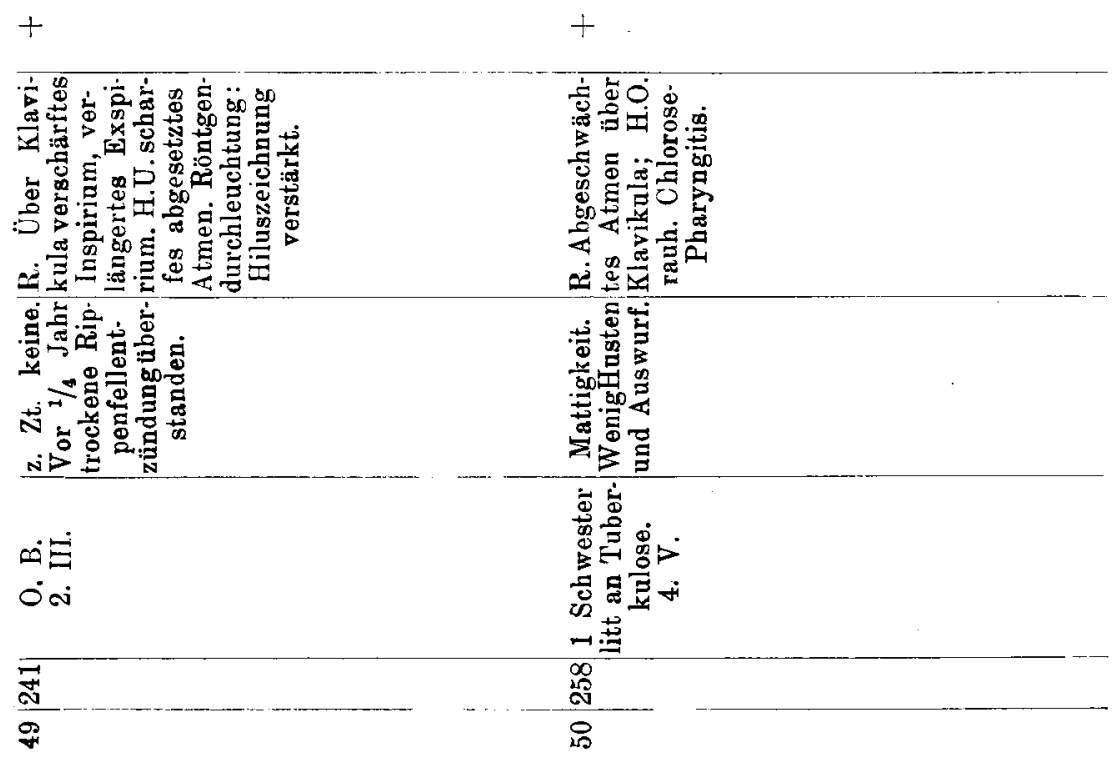




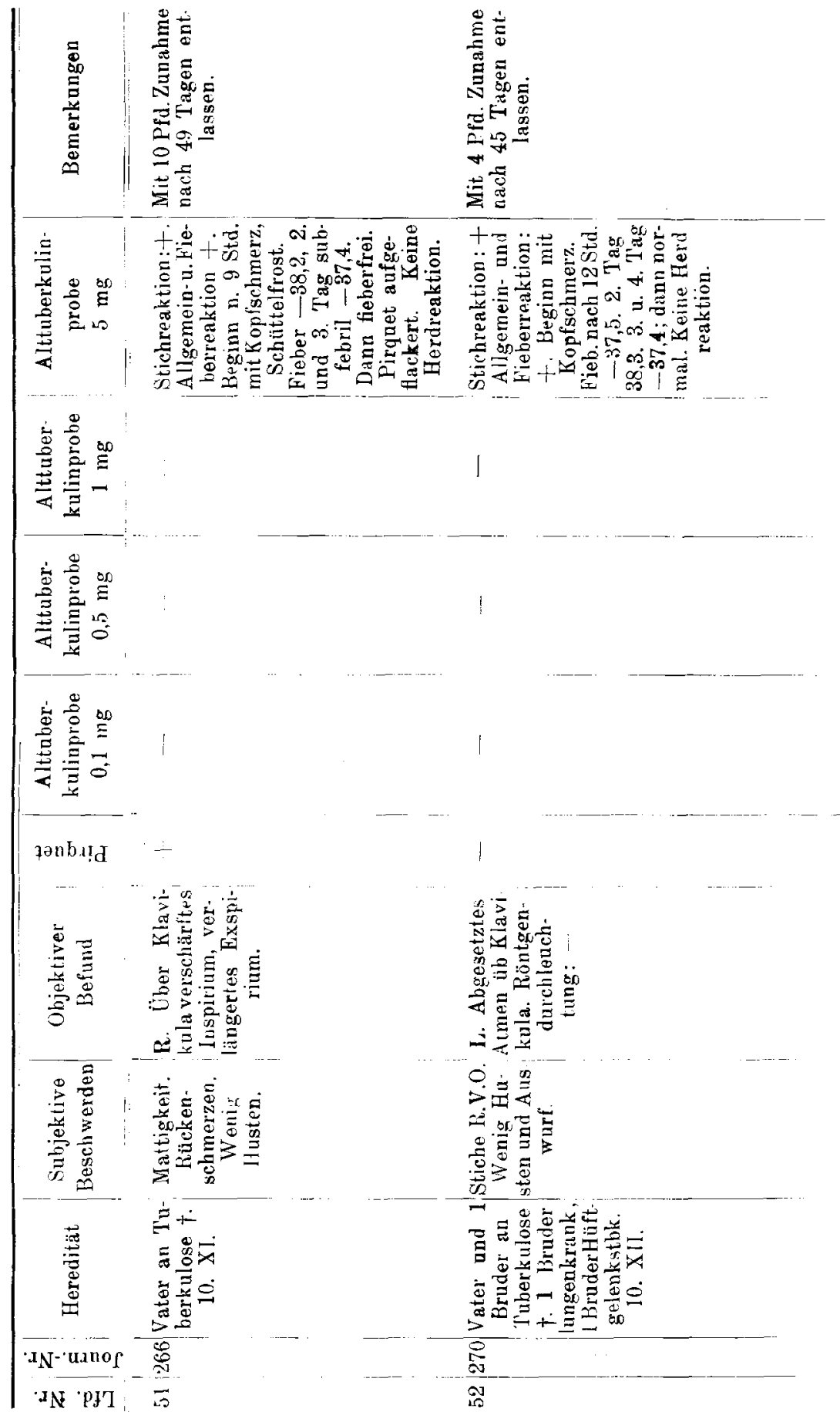



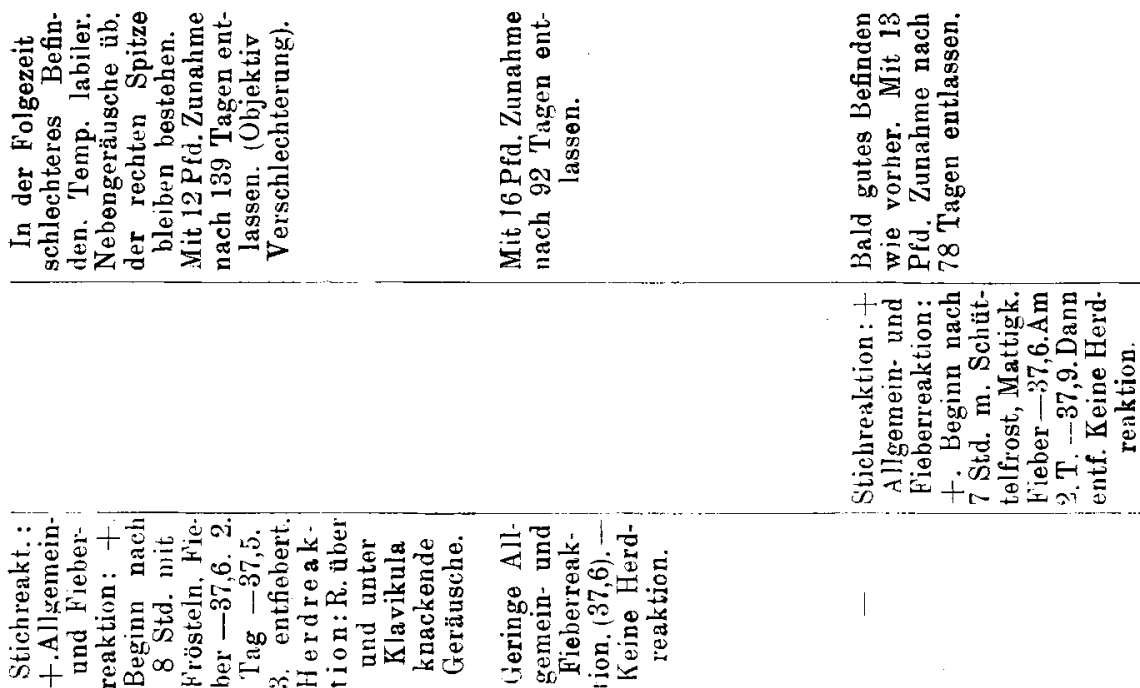

$+$

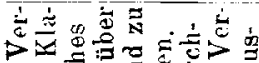

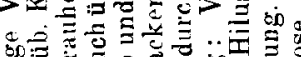

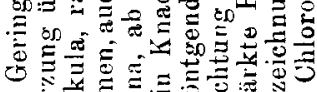

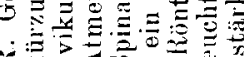
过定>

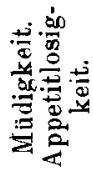

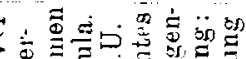

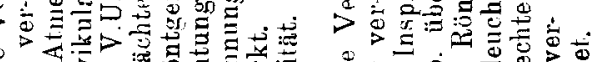
का

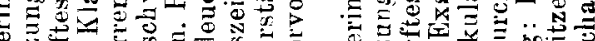

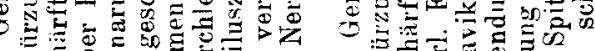

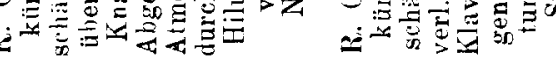

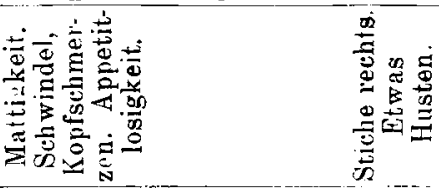

$\dot{0} \dot{0}$
$\dot{0} \dot{0}$

范

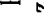
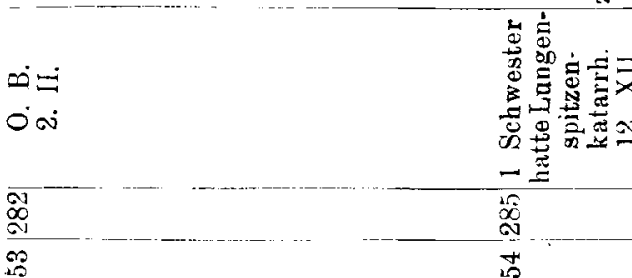

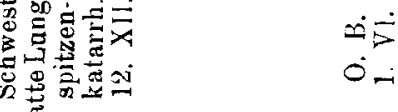

$-\pi$ 
Wie aus den Tabellen ersichtlich, handelt es sich um sogenannte zweifelhafte Fälle; sie waren meist schon durch die Hände von zwei Untersuchern gegangen und von dem behandelnden und dem Vertrauensarzt oft auf Grund einer einzigen Untersuchung als lungenkrank bezeichnet und mit der Diagnose Lungenspitzenkatarrh oder beginnende Tuberkulose (I. Stadium) eingewiesen. Gewiss auch wir hatten bei der Aufnahme, wie aus der Zusammenstellung hervorgeht, die geringfügigen Veränderungen, meist über der rechten Lungenspitze, gefunden. Eine geringe Schallabschwächung, die besonders rechts oft normal ist und geringe Veränderungen des Atemgeräusches sind aber noch nicht, wie meist ohne weiteres angenommen war, gleichbedeutend mit aktiver, $d$. h. behandlungsbedürftiger Tuberkulose: und nur aktive Tuberkulosen gehören in die Heilstätte. Allerdings. sind, wie Ritter betont, die üblichen für den Charakter der Tuberkulose entstandenen Bezeichnungen, wie latent, manifest, aktiv, inaktiv, behandlungsbedürftig und nicht behandlungsbedürftig sehr dehnbare Begriffe und zum Teil von dem persönlichen Ermessen und der Fähigkeit des Untersuchers abhängig; wir müssen aber mit ihnen rechnen und insbesondere der Begriff der Aktivität und der dadurch bedingten Behandlungsbedürftigkeit besitzt praktischen Wert. Wir konnten in unseren 55 Fällen nach 14 tägiger Beobachtung nicht mit Sicherheit eine aktive Tuberkulose feststellen und nahmen daher bei ihnen die subkutane Alttuberkulinprobe vor, um zu sehen, wie weit sie uns Aufschluss über die Aktivität oder Inaktivität des Prozesses geben würde. Die Krankengeschichte ist in den Tabellen nur im Auszuge in ihren für unsere Frage wichtigsten Punkten wiedergegeben, bei der Vorgeschichte insbesondere die hereditäre und Brehmers Belastung, nach dem in kinderreichen Familien besonders die jüngsten Kinder für Tuberkulose disponiert sind. Die hereditäre Belastung wird auch vielfach ohne objektiven Befund für die Diagnose einer tuberkulösen Erkrankung bei Erwachsenen zu hoch bewertet. In unseren Fällen wurde $\mathbf{3 4} \mathrm{mal}$ in der Vorgeschichte keine Tuberkulose in Familie oder Verwandtschaft angegeben, $21 \mathrm{mal}$ wurde die Frage bejaht; oft waren es aber ganz entfernte Verwandte oder nähere, mit denen die Patienten aber gar nicht oder nur selten in Berührung gekommen waren. Brehmers Belastung - vom 4. Kinde ab gerechnet - lag in 24 Fällen vor. Auch die Klagen der Patienten werden mitunter zu sehr berücksichtigt: Mattigkeit, Stiche zwischen den Schulterblättern, „Rückenschmerzen“, selbst angebliche Nachtschweisse und „Blutstreifen“ im Auswurf, Beschwerden, die so häufig in der Vorgeschichte wiederkehren und vielfach ohne objektiven Lungenbefund entscheidend für die Diagnose verwertet 
werden, sollten nicht nur bei Lungenkranken angenommen werden, sie kommen auch ohne Lungenerkrankung vor. Es sollte keine „subjektive" wie so häufig, sondern eine objektive aktive Lungentuberkulose diagnostiziert werden. Wir finden in unseren Fällen diese allgemeinen subjektiven Beschwerden, die den Patienten zum Arzt führten, fast stets. Auch Husten wurde 26 mal, meist ohne Auswurf, angegeben; wenn Auswurf vorhanden war, stammte er aber vom Rachen bei gleichzeitig bestehender Pharyngitis, die häufig nachgewiesen werden konnte, wie auch der Husten oft durch Pharyngitis bedingt war; in 29 Fällen waren Husten und Auswurf überhaupt nicht vorhanden. Dass der positive Pirquet für die Aktivität des Prozesses bei Erwachsenen und schon bei älteren Kindern gar nichts beweist, sondern nur die Tuberkuloseinfektion anzeigt, sollte allge. mein bekannt sein und doch wird noch oft betont, dass die Diagnose durch Pirquet "gesichert" ist und erst kürzlich las ich wieder in einem Attest bei unklarem Lungenbefunde: „Es wurde die Pir quetsche Probe ausgeführt, die deutlich positiv war; ich stelle die Diagnose "aktive Lungentuberkulose“.

Betrachten wir nun die Ergebnisse der Alttuberkulinprobe: In 7 Fällen (Nr. 9, 12, 21, 26, 27, 38, 43) war bis $5 \mathrm{mg}$ keine Reaktion aufgetreten; es wurde hierbei von einigen wohl über Schmerzen an der Stichstelle geklagt, aber eine deutliche Stichreaktion, die sich durch Rötung und Schwellung der Impfstelle zu erkennen gibt, wurde in keinem Falle festgestellt; ebensowenig trat eine Allgemein-Fieberoder Herdreaktion auf; es war somit erwiesen, dass hier keine aktive Tuberkulose vorlag und in den 5 Fällen (Nr. 9, 21, 26, 38, 43), in denen der vorher angestellte Pirquet schon negativ war, bestand wohl überhaupt keine Beziehung zur Tuberkulose, während im Falle 12 und 27 der positive Pirquet nur die tuberkulöse Infektion anzeigte. Die Einspritzungen hatten keine Störungen auf das Befinden der Patientinnen ausgeübt; in einem Falle wurde nach der Probe sogar nicht mehr so über Mattigkeit geklagt wie vorher. Die subjektiven. Beschwerden waren somit nicht auf die Lunge zurückzufübren, sondern durch Nervosität, Chlorose oder Pharyngitis bedingt. Gewiss, die Patienten waren erholungsbedürftig und aus diesem Grunde be. hielten wir sie meist länger - wenn auch nicht immer volle 3 Monate -, aber in eine Heilstätte gehören sie nicht. Später wurden derartige Fälle, die übrigens seit Kriegsausbruch seltener wurden, da sich seitdem meist nur wirklich Lungenkranke zur Kur meldeten und auch die Zahl der offenen Tuberkulosen häufte, in ein Erholungsheim verlegt.

In 48 Fällen war die subkutane Alttuberkulinprobe, wie die $\mathrm{Zu}$ - 
sammenstellung zeigt, positiv gewesen, aber nur 12 mal konnten dabei auf Grund sorgfältiger, $z u$ den verschiedenen Zeiten vorgenommener Untersuchungen sichere Herdreaktionen nachgewiesen werden, in den anderen 36 Fällen wurde ausser der meist vorhandenen Stichreaktion nur eine Allgemein- und Fieberreaktion beobachtet, die fast immer zusammen vorkommt. Die Allgemeinreaktion, die dem Auftreten von Fieber etwas vorangeht, beginnt frühestens nach 6 , meist nach $10-12$ Stunden mit dem Gefühl des Unbehagens, Kopischmerzen, Übelkeit, mitunter kommt es sogar zum Erbrechen; auch Schüttelfrost wurde beobachtet, wie überhaupt in einigen Fällen ein schweres Kranklieitsgefühl und elendes Befinden, allerdings meist nur für einige Stunden, bestand. Fieber tritt etwas später hinzu, geht mitunter plötzlich in die Höhe, oft aber allmählich und erreicht nach 24 Stunden meist den höclısten Stand; die Temperatur hält sich am 2. Tage in geringerer Lrhebung und kehrt meist am 3. Tage zur Norm zurück, ist dann in einigen Fällen sogar niedriger und gleichmässiger als vor den Injektionen. Spätere Fiebersteigerungen, nach dem 2. Tage, sind selten, kommen aber vor, so in einem unserer Fälle (Nr. 49) erst nach 52 Stunden. Temperaturen über 39 wurden 8 mal beobachtet, meist zwischen 38 und $39^{\circ}$, selten unter 38 , dann aber mindestens $0.5^{0}$ höher als früher. Fiebersteigerungen, die nicht mindestens mehrere Stunden anhalten, sind nicht zu verwerten. Die Allgemein- und Fieberreaktionen wurden beobachtet: bei $0,1 \mathrm{mg} 2 \mathrm{mal}$ ( $\mathrm{Nr}$. 20 u. 44); bei $0.5 \mathrm{mg} 7 \mathrm{mal}$ (Nr. 13, 15, 17, 34. 36, 37, 40, 49); bei $1 \mathrm{mg}$ $14 \mathrm{mal}$ (Nr. 1, 4, 5. 6, 7, 24, 29. 31, 32, 33, 45. 46, 50, 54); bei $5 \mathrm{mg} 13 \mathrm{mal}$ (Nr. 2, 11, 17, 19, 28, 35, 39, 41, 47, 48, 51, 52, 55). Die ron manchen Autoren betonte "Tatsache", dass Reaktionen auf kleine Dosen eine gewisse klinische Bedeutung haben und einen aktiven Prozess anzeigen, und zwar um so aktiver, je kleiner die Dosis und je stärker und rascher die Reaktion erfolgt, kann durch unsere Untersuchungen nicht bestätigt werden. Die Allgemein- und Fieberreaktion ist für die Entscheidung der Frage der Aktivität nicht zu verwerten, sie zeigt wie die Kutanprobe nur das C̈berstehen einer tuberkulösen Infektion an. Schädigungen haben wir durch die Allgemein- und Fieberreaktion nie auftreten sehen; die Reaktion hatte sogar in verschiedenen Fällen einen günstigen Eintluss aut die subjektiven Beschwerden, es wurde wiederholt nach der Reaktion das Allgemeinbefinden besser als ror den Einspritzungen.

In nur 12 Fällen wurde also eine sichere Herdreaktion nachgewiesen, die sich durch Auftreten von frïher nicht gehörten Rasselgeräuschen und nur selten durch deutliche Änderung des Atemgeräusches zu erkennen gab. Eine Veränderung des Perkussionsbe- 
fundes, in dem Sinne, dass „Dämpfungen“ durch die Reaktion hervorgerufen wurden, wie es merkwürdigerweise Romberg angibt, haben wir nie beobachtet. Die Herdreaktion kam vor: bei $0,1 \mathrm{mg}$ $1 \mathrm{mal}$ (Nr. 10); bei $0,5 \mathrm{mg} 1 \mathrm{mal}$ (Nr. 8); bei $1 \mathrm{mg} 4 \mathrm{mal} \mathrm{(Nr.} \mathrm{16,} \mathrm{25,}$ 30,53 ); bei $5 \mathrm{mg} 6 \mathrm{mal}$ (Nr. 3, 14, 18, 22, 23, 42). In einem Falle wurde eine Herdreaktion ohne Allgemein- und Fieberreaktion beobachtet (Nr. 23), sonst war stets auch eine Allgemein- und Fieberreaktion vorhanden. Durch die Herdreaktion wurde 6 mal eine vorübergehende Schädigung von kürzerer oder längerer Dauer herrorgerufen. In Fall 3 wurde eine bis dahin geschlossene Form durch die Herdreaktion vorübergehend zu einer offenen gemacht und die Patientin in ihrer Kur so zurückgebracht, dass noch eine Kurverlängerung erforderlich wurde, bis der Schaden beseitigt war; auch im Fall 53 trat eine längere Schädigung durch Verschlechternng des Allgemeinbefindens, labilere Temperatur auf, und die durch die Herdreaktion ausgelösten Rasselgeräusche waren auch bei der Entlassung noch zu hören, wie auch in den Fällen Nr. 10, 16, 23 die Rasselgeräusche bis zur Entlassung bestehen blieben. In Fall 14 blieb die früher normale Temperatur nach der Herdreaktion subfebril und die subjektiven Beschwerden verstärkten sich. In den Fällen 8 und 30 verschwanden die durch die Herdreaktion hervorgetretenen Rasselgeräusche nach wenigen Tagen und das subjektive Befinden wurde sogar besser als vorher; in den anderen Fällen $(18,22,25,42)$ war das Befinden bald wieder wie vor der Reaktion und die Geräusche gingen gleichfalls zurück. Alle Fälle, bei denen die subkutane Probe angestellt war, konnten aber mit meist erheblicher - durch die Liegekur bedingte - Gewichtszunahme als berufsfähig entlassen werden; nur Fall 53 blieb nur teilweise berufsfähig. Das Prädikat "geheilt" vermeide ich überhaupt, da die Heilung einer aktiven Tuberkulose nach unserer Ansicht nicht durch eine Heilstättenkur erzielt werden kann, sondern sich erst nach Jahren in der Arbeit erkennen lässt; lediglich aber zur Verbesserung der Statistik inaktive Fälle, die auch vor der. Aufnahme schon "geheilt" waren, als geheilt bei der Entlassung zu bezeichnen, ist nicht angängig.

Viel leistet jedenfalls die subkutane Alttuberkulinprobe gewiss nicht zur Erkennung der aktiven Lungentuberkulose bei Erwachsenen,

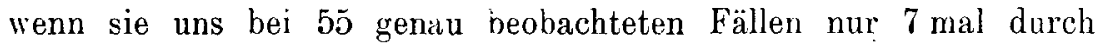
ihren negativen Ausfall sichere Aufklärung verschaffte, in den anderen 48 positiven Fälien nur $12 \mathrm{mal}(25 \%)$ Herdreaktionen auslöst, von denen 6 vorübergehend geschädigt wurden und es ïberhaupt fraglich blieb, ob der Prozess durch die Herdreaktion nicht erst aktiv geworden war. Der Ansicht vieler Autoren, dass die Herdreaktion das 
Zeichen „unbedingter Aktivität“ ist, kann nicht ohne weiteres beigepflichtet werden, wir haben Herdreaktionen auch nicht, wie Roepke und Bandelier, bei einem hohen Prozentsatz nachweisen können und trotz Anwendung "lege artis" vorübergehende Schädigungen gesehen. Ich stimme Ritter bei, dass der nachweisbare Eintritt einer deutlichen Herdreaktion durchaus unregelmässig und unabhängig von klinischer Alitivität und Inaktivität ist. „Dieselbe Unsicherheit, die bei der klinischen Diagnose in den zweifelhaften Fällen uns zur Ausfübrung der Subkutanprobe veranlasste, blieb auch meist bei der sperifischen Diagnose nach der positiven Reaktion bestehen". Wir können das Tuberkulin daher zur Diagnose entbehren, um so mehr als dem Patienten durch die Reaktion mitunter einige Tage höchst elenden Befindens bereitet werden. Die genaue Untersuchung - physikalische und Röntgenkontrolle - insbesondere die längere Beobachtung wird doch meist erkennen lassen, ob die subjektiven Beschwerden, auf die allein vielfach $\mathrm{zu}$ grosses Gewicht gelegt wird, von einer aktiven Tuberkulose herrühren, oder eine andere Ursache haben. Es gehört zweifellos, wie Lorey mit Recht sagt, zu den allerschwierigsten und verantwortungsreichsten Aufgaben des Arztes, eine beginnende Tuberkulose festzustellen oder in Verdachtsfällen mit Sicherheit auszuschliessen, und die Diagnose darf nicht leichtfertig in so zweifelhaften Fällen auf Grund einer Untersuchung gestellt werden. Ein Urteil über die Behandlungsbedürftigkeit unklarer Fälle schon auf Grund einer Untersuchung abzugeben, wie es oft geschieht, ist gar nicht möglich, und auch durchaus nicht nötig. Derartige Fälle sind entweder einer Beobachtungsstation, wie sie in vielen Heilstätten ist, zu überweisen - aber nicht schon als Lungenkranke (!) - oder, da die Ruhebeobachtuug in der Anstalt, wie aus unseren Fällen ersichtlich, die Frage der Aktivität oder Inaktivität schwieriger löst, zweckmässiger in ihrem Beruf zu belassen und bei der Arbeit zu beobachten, wie es auch Ritter vorschlägt, unter genauer Kontrolle der Temperatur, des Körpergewichtes, wiederholter Lungenuntersuchungen, eventuell Röntgenaufnahme (Übersicht- und Spitzenaufnahme). Geschadet kann durch die Fortsetzung der Arbeit in solchen Fällen niemals werden, und ein geübter Untersucher wird dann am schnellsten zur Klarheit kommen, ob ein aktiver Prozess vorliegt und Behandlung bedingt und dann die Überweisung in die Heilstätte vornehmen. 


\section{Literatur.}

1. Bandelier-Roepke, Die Klinik der Tuberkulose.

2. Dieselben, Lehrbuch der spezifischen Diagnostik und Therapie der Tuberkulose.

3. Lorey, Das Röntgenverfahren bei der Lungentuberkulose: Brauer. Schröder-Blumenfeld, Handbuch der Tuberkulose 1915. Bd. 1.

4. Ritter, Die klinische Bedeutung der Tuberkulinreaktionen: BrauerSchröder-Bl umenfeld, Handbuch der Tuberkulose 1915. Bd. 1.

5. Otten, Mediz. Klinik 1910.

6. Romberg, Verhandl. d. 27. Kongr. f. innere Med. 1910.

7. Pannwitz, Tuberkulin in Deutschland. Bericht für die XI. internationale Tuberkulose-Konferenz. Berlin 1913.

8. Sehröder, Über Tuberkulinbehandlung. Beitr. z. Kl. d. Tuberk. 1912. Bd. 23. H. 1.

9. Ulrici, Zur Frage der ambulanten Anwendung des Tuberkulins. Beitr. z. Kl. d. Tuberk. 1912. III. Sappl.-Bd.

10. Walterhöfer, Beitrag zur Klinik der Tuberkulose. Bd. 21.

11. Weihrauch, Beitrag zur Klinik der Tuberkulose. Bd. 15. 